\title{
Additivity of the Entropy and Definition of the Temperature for Quantum Systems
}

\author{
H. Roos \\ Institute for Theoretical Physics, Groningen, The Netherlands*
}

Received April 12; in revised form July 27, 1973

\begin{abstract}
A closed quantum system $\mathscr{S}$ is considered which is described by a microcanonical ensemble. $\mathscr{S}$ consists of two rather weakly interacting subsystems $\mathscr{S}_{1}, \mathscr{S}_{2}$. In a rigorous way, the additivity of the entropy is proved by deriving an expression for the entropy density of $\mathscr{S}$ in terms of the entropy densities of $\mathscr{S}_{1}$ and $\mathscr{S}_{2}$. "Rigorous" implies that the thermodynamic limit is taken. In the second part, it is shown how a microcanonical state $\omega_{\varepsilon}(\Lambda)=\lim _{\Lambda \rightarrow \infty} \frac{\operatorname{Tr} \delta^{A}(H(\Lambda)-E) A}{\operatorname{Tr} \delta^{A}(H(\Lambda)-E)}$ of the composite system - provided this limit exists - gives rise to a "canonical" state $\omega^{\beta}$, when restricted to $\mathscr{S}_{1}$, provided $\mathscr{S}_{1}$ is very "small" as compared to $\mathscr{S}_{2} ; \omega^{\beta}$ is defined as a limit of Gibbs states. This yields a definition of the equilibrium temperature $\beta^{-1}$.
\end{abstract}

\section{Introduction}

The concept of temperature can be introduced in statistical mechanics in several ways. Up to now there does not exist a general proof of the equality of time averages and ensemble averages of observables; it has to be taken as an axiom that there are suitable ensembles. One can start with canonical or grand canonical ensembles with partition functions depending on a parameter $\beta$ and show that everything works if $\beta$ is the inverse temperature. A more satisfactory way of introducing the temperature is to consider several microcanonical ensembles representing closed systems, provide them with a - however weak - thermal interaction and show that there is a parameter govering the equilibrium between them, which is to be defined as the temperature (or a function thereof). A third possibility is, to deal with a "large" system in thermal contact with a "small" system and show that the "small" one can be described by a canonical ensemble with a parameter $\beta$ depending only on the "large" system, the heat reservoir. This is well known; in this paper, we aim at giving new proofs considering quantum systems from the very beginning and working in the context of rigorous statistical mechanics as developed by Ruelle and many others.

* On leave of absence from the Institut für Theoretische Physik, Universität Göttingen. 
Our starting point is the following axiom:

$\left(\mathrm{A}_{0}\right)$ A closed system in equilibrium is described by a microcanonical ensemble which is defined by the requirement that all energetically allowed quantum states of the system have equal probability.

(Normally, by using the word "states", we mean states over an algebra of observables, but here, of course, we mean eigenstates of the Hamiltonian of the system.) We shall specify this axiom in the next sections because we want to perform the thermodynamic limit.

As a first step, we have to prove the additivity of the entropy, i.e., prove that the whole entropy of several systems in thermal equilibrium with each other is the sum of the entropies (apart from mixing entropy) of the systems, provided the thermal interaction between them is very weak. Since we are going to take the thermodynamic limit, we shall give a formula for the entropy density of the compound system as an appropriate sum of the entropy densities of its members. This will be done in Section II. In the third section, we shall give a first definition of the temperature based on the result of Section II, and prove some lemmas which are necessary for establishing our main theorem in Section IV, where we shall show, starting with a microcanonical state for the compound system, that the "small" system $\mathscr{S}_{1}$ can be described by a limit $\Lambda \rightarrow \infty$ of Gibbs states $\omega_{A}^{\xi_{\Lambda}}$, defined by density operators

$$
e^{-\xi_{\Lambda} H_{1}(\Lambda)} / \operatorname{Tr} e^{-\xi_{\Lambda} H_{1}(\Lambda)},
$$

where $H_{1}(\Lambda)$ are the local Hamiltonians of the regions $\Lambda$, describing $\mathscr{S}_{1}$. Section $\mathrm{V}$ contains supplementary remarks.

\section{Additivity of the Entropy}

We consider a system $\mathscr{S}$ composed of two subsystems $\mathscr{S}_{1}$ and $\mathscr{S}_{2}$ defined as thermodynamic limits of sequences of finite systems $\mathscr{S}\left(\Lambda_{1}\right)$, $\mathscr{S}\left(\Lambda_{2}\right)$ and $\mathscr{S}(\Lambda)$, respectively, with finite regions $\Lambda_{i}$,

$$
\Lambda_{1} \cap \Lambda_{2}=\emptyset, \quad \Lambda_{1} \cup \Lambda_{2}=\Lambda .
$$

The finite systems are described by Hamiltonians $H_{i}\left(\Lambda_{i}, n_{i}\right), i=1,2$, and $H(\Lambda, n)$, where

$$
H(\Lambda, n)=H_{1}\left(\Lambda_{1}, n_{1}\right)+H_{2}\left(\Lambda_{2}, n_{2}\right)+\lambda W(\Lambda, n),
$$

operating in a Hilbert space $\mathscr{H}(\Lambda)=\mathscr{H}_{1}\left(\Lambda_{1}\right) \otimes \mathscr{H}_{2}\left(\Lambda_{2}\right) \cdot n_{1}$ and $n_{2}$ denote the number of particles in $\mathscr{S}_{1}$ and $\mathscr{S}_{2}$,

$$
n=n_{1}+n_{2} \text {. }
$$

The $H_{i}$ are of the form $\hat{H}_{1} \otimes 1$ and $1 \otimes \hat{H}_{2}$, respectively. 
Our basic statistical assumption is the following specification of the axiom $\left(\mathrm{A}_{0}\right)$ :

$\left(A_{1}\right)$ The entropy density of a closed system in thermodynamic equilibrium with density $\varrho$ and energy density $\varepsilon$ is given by

$$
s(\varrho, \varepsilon)=\lim _{\Lambda \rightarrow \infty} V(\Lambda)^{-1} S(\Lambda, n, E)
$$

whenever this limit exists; here "lim" means $\Lambda \rightarrow \infty$ in the sense of Fisher [1], with $V(\Lambda)^{-1} n \rightarrow \varrho, V(\Lambda)^{-1} E \rightarrow \varepsilon$, where

$$
\begin{aligned}
V(\Lambda) & =\text { volume of the region } \Lambda, \\
S(\Lambda, n, E) & =\log \Omega^{-}(\Lambda, n, E), \\
\Omega^{-}(\Lambda, n, E) & =\text { number of eigenvalues of } H(\Lambda, n) \text { below } E \\
& =\operatorname{Tr} \delta^{-}(H(\Lambda, n)-E), \\
\delta^{-}(x) & =\left\{\begin{array}{lr}
1 & -\infty<x<0 \\
0 & x>0
\end{array}\right.
\end{aligned}
$$

According to Ruelle [2], we can as well define $S(\Lambda, n, E)$ by

$$
\log \Omega^{\Delta}(\Lambda, n, E)=\log \operatorname{Tr} \delta^{\Delta}(H-E), \delta^{\Delta}(x)=\left\{\begin{array}{ll}
1 & 0<x<\Delta \\
0 & \text { elsewhere }
\end{array},\right.
$$

without affecting the result of the limiting procedure.

We have to make further assumptions in order to ensure the existence of the above limit for the systems $\mathscr{S}_{1}, \mathscr{S}_{2}$, and $\mathscr{S}$ :

(B) $H_{i}\left(\Lambda_{i}, n_{i}\right), i=1,2$, are defined by stable and tempered potentials:

$$
H_{i}\left(\Lambda_{i}, n_{i}\right)=\text { Friedrichs extension of }\left(T_{i}+U_{i}\right),
$$

where the kinetic energy may be represented in Hilbert spaces $\mathscr{H}_{i}\left(\Lambda_{i}\right)$ of suitably symmetrized or antisymmetrized square integrable functions (cf. [2]) by

$$
T_{i}=\sum_{k=1}^{n_{i}}\left(-\frac{1}{2 m_{i}} \Delta_{k}\right), \quad \Delta_{k}=\text { Laplace operators, }
$$

the potential energy $U_{i}$ by stable, tempered potentials,

$$
U_{i}\left(x_{1} \ldots x_{n_{1}}\right)=\sum_{k \geqq 2} \sum_{1 \leqq J_{1} \leqq J \leqq \ldots j_{k} \leqq n_{i}} \phi^{k}\left(x_{J_{1}} \ldots x_{J_{k}}\right) ; \quad x_{\alpha} \in \Lambda_{i} .
$$

(C) $W$ is given by tempered potentials in such a way that

(i) $W$ is a symmetric operator,

(ii) $U_{1}+U_{2}+W$ is stable,

(iii) $\mathscr{D}\left(U_{1}+U_{2}\right) \subset \mathscr{D}(W)$, where $\mathscr{D}(A)$ denotes the domain of definition of $A$. 
(D) The potentials defining $U_{i}$ and $W$ are translationally invariant. According to Ruelle, (B) is sufficient to guarantee the existence of $s_{1}\left(\varrho_{1}, \varepsilon_{1}\right)$ and $s_{2}\left(\varrho_{2}, \varepsilon_{2}\right)$ as defined in $\left(\mathrm{A}_{1}\right)$, if $\Lambda_{i} \rightarrow \infty$ (Fisher),

$$
n_{i} V\left(\Lambda_{i}\right)^{-1} \rightarrow \varrho_{i}, E_{i} V\left(\Lambda_{i}\right)^{-1} \rightarrow \varepsilon_{i}, i=1,2,
$$

provided $\varrho_{i}$ and $\varepsilon_{i}$ have suitable values. To be more precise, the density has to be $\geqq 0$ and smaller than some closest packing density, which may be finite or infinite, depending on the potentials; $\varepsilon_{i}$ have to be larger than $\varepsilon_{i 0}(\varrho), i=1,2$, respectively, where

$$
\begin{aligned}
\varepsilon_{i 0}(\varrho) & =\lim V(\Lambda)^{-1} E_{i 0}\left(\Lambda_{i}, n_{i}\right), \\
E_{i 0} & =\text { ground state energy of } H_{i}\left(\Lambda_{i}, n_{i}\right) .
\end{aligned}
$$

According to Ruelle, these limits exist. Whenever we speak of "suitable" densities, we refer to these conditions.

In Appendix A, we shall give two examples of how $(C)$ can be fulfilled. Due to $(\mathrm{C})$ we can define $H(\Lambda, n)$ as the Friedrichs extension of $H_{1}\left(\Lambda_{1}, n_{1}\right)+H_{2}\left(\Lambda_{2}, n_{2}\right)+\lambda W(\Lambda, n)$. Denote the number of eigenvalues of $H$ below $E$ by $\Omega_{\lambda}^{-}(\Lambda, n, E)$. We are interested in the limit $\Lambda \rightarrow \infty$ which, in the following, always means

$$
\begin{gathered}
\Lambda_{1}, \Lambda_{2} \rightarrow \infty \text { in the sense of Fisher, maintaining condition (1); } \\
\Lambda=\Lambda_{1} \cup \Lambda_{2} \rightarrow \infty \text { in the sense of Fisher ; } \\
V\left(\Lambda_{i}\right) / V(\Lambda)=\kappa_{i}, \kappa_{i}=\text { const }>0, \kappa_{1}+\kappa_{2}=1 ; \\
n_{i} / V\left(\Lambda_{i}\right) \rightarrow \varrho \text {, and thus } n / V(\Lambda)=n_{1}+n_{2} / V\left(\Lambda_{1}\right)+V\left(\Lambda_{2}\right) \rightarrow \varrho ; \\
E / V(\Lambda) \rightarrow \varepsilon .
\end{gathered}
$$

(As to the case of different $\varrho_{1}$ and $\varrho_{2}$ and to the case of intersecting region $\Lambda_{i}$, see Remark 2.6 at the end of this section.)

Assumption (D) is needed so that (5a) can be fulfilled. Given (5a), a necessary and sufficient condition to guarantee $(5 b)$ is the following:

$$
\Delta\left(\Lambda_{1}, \Lambda_{2}\right)=\inf _{x \in \Lambda_{1}, y \in \Lambda_{2}}|x-y| \leqq \text { const } \cdot\left(d\left(\Lambda_{1}\right)+d\left(\Lambda_{2}\right)\right),
$$

where $d\left(\Lambda_{i}\right)$ are the diameters of $\Lambda_{i}, i=1,2$ (see Appendix E).

Proposition 2.1. Assume ( $\left.\mathrm{A}_{1}\right),(\mathrm{B}),(\mathrm{C})$, (D); then

(i) for all $\lambda \leqq 1$ and for suitable $\varrho$, there exists the thermodynamic limit

$$
s_{\lambda}(\varrho, \varepsilon)=\lim _{\Lambda \rightarrow \infty} V(\Lambda)^{-1} \log \Omega_{\lambda}^{-}(\Lambda, n, E) ;
$$

(ii) $\lim _{\lambda \rightarrow 0} \lim _{\Lambda \rightarrow \infty} V^{-1} \log \Omega_{\lambda}^{-}(\Lambda, n, E)=\lim _{\Lambda \rightarrow \infty} \lim _{\lambda \rightarrow 0} V^{-1} \log \Omega_{\lambda}^{-}(\Lambda, n, E)$. 
As long as we are not interested in problems of the approach to equilibrium, we may consider arbitrarily weak thermal interactions, i.e., arbitrarily small $\lambda$. Furthermore, it is clear that we cannot expect strict additivity of the entropies unless we let the interaction vanish; hence our interest in the second part of the above proposition.

Proof. Due to (B) and (C), $U_{1}, U_{2}$, and $U_{1}+U_{2}+W$ are stable:

$$
U_{1} \geqq-n_{1} B_{1}, \quad U_{2} \geqq-n_{2} B_{2}, \quad U_{1}+U_{2}+W \geqq-n B .
$$

With $B_{0}=\max \left(\left|B_{1}\right|,\left|B_{2}\right|,|B|\right)$ then follows

$$
\begin{aligned}
U_{1}+U_{2}+\lambda W & =(1-\lambda)\left(U_{1}+U_{2}\right)+\lambda\left(U_{1}+U_{2}+W\right) \\
& \geqq-(1-\lambda)\left(n_{1}+n_{2}\right) B_{0}-\lambda n B_{0}=-n B_{0},
\end{aligned}
$$

the lower bound being independent of $\lambda$. Hence $H(\Lambda, n)$ fulfills the same conditions as the $H_{i}$, thus we have only to cite Ruelle [2], in order to establish (i). The proof of (ii) is accomplished by a close inspection of Ruelle's proof of the existence of the limit. It rests on the stability and temperedness of the interaction. The corresponding inequalities for $U_{\lambda}=U_{1}+U_{2}+\lambda W$ are fulfilled uniformly in $\lambda$, i.e., the constants appearing in those inequalities are independent of $\lambda$ for $\lambda<1$. This is trivial as far as the temperedness is concerned and, as to the stability, it is ensured by (C) (ii). Looking carefully through Ruelle's proof, one realizes that everything goes through uniformly in $\lambda$, since the only other inequalities which appear are estimates for quantities like $E\left(\Lambda_{0}, 1, \sigma\right)$, $E\left(\Lambda_{0}, 0, \sigma\right), E\left(\Lambda_{N}, 1, \log V\left(\Lambda_{N}\right)\right)$, referring to zero- or one-particle expressions; clearly, the latter are defined independent of any interaction. Hence we can interchange the limits $\lambda \rightarrow 0$ and $\Lambda \rightarrow \infty$ Q.E.D.

Remark 2.2. (B) and (C) are more or less technical assumptions which we do not want to put much emphasis upon. (B) is not a necessary condition for the existence of the limit $s(\varrho, \varepsilon)$. Given any proof of the existence of this limit, one has to modify (C) appropriately in order to make sure that the limit for the composed system exists uniformly in $\lambda$. It is to be expected that this can always be achieved, for instance, by the use of a thermal interaction $W$ which is given by the same type of potentials as those defining $U_{1}$ and $U_{2}$.

Due to Proposition 2.1 , we can study $\lim _{\Lambda \rightarrow \infty} V(\Lambda)^{-1} \log \Omega_{0}^{-}(\Lambda, n, E)$ instead of $\lim _{\lambda \rightarrow 0} s_{\lambda}(\varrho, \varepsilon)$, which enables us to prove the following

Theorem 2.3. Let us assume $\left(\mathrm{A}_{1}\right)$ and make further appropriate assumptions which guarantee the validity of (i) and (ii) of Proposition 2.1. Then, with the definitions (5) to (8), we have

$$
\lim _{\lambda \rightarrow 0} s_{\lambda}(\varrho, \varepsilon)=\sup _{\varepsilon^{\prime} \in I(\varepsilon)}\left[\kappa_{1} s_{1}\left(\varrho, \frac{\varepsilon^{\prime}}{\kappa_{1}}\right)+\kappa_{2} s_{2}\left(\varrho, \frac{\varepsilon-\varepsilon^{\prime}}{\kappa_{2}}\right)\right],
$$


with

$$
I(\varepsilon)=\left[\kappa_{1} \varepsilon_{10}(\varrho), \varepsilon-\kappa_{2} \varepsilon_{20}(\varrho)\right] .
$$

The $\varepsilon_{i 0}$ are defined in (4). Again, $\varepsilon$ and $\varrho$ have to be chosen suitably.

Proof. We want to compute the number $\Omega_{0}^{-}(\Lambda, n, E)$ of eigenvalues of $H_{0}(\Lambda, n)=H_{1}\left(\Lambda_{1}, n_{1}\right)+H_{2}\left(\Lambda_{2}, n_{2}\right)$. These eigenvalues are sums of eigenvalues $E_{1 r}$ and $E_{2 s}$ of $H_{1}$ and $H_{2}$. In the following, we shall suppress the arguments $\Lambda, n$ and $\Lambda_{i}, n_{i}$. For fixed $E$, we can find a $\delta>0$ such that the eigenvalues $E_{1 r}$ which are smaller than $E$, differ from each other by more than $\delta$. Define

$$
E^{k}=E_{10}+k \delta, \quad k=1,2, \ldots,
$$

then there is at most one eigenvalue $E_{1 r}$ between $E^{k}$ and $E^{k-1}$; and we can write $\Omega_{0}^{-}(E)$ as

$$
\Omega_{0}^{-}(E)=\sum_{k}\left(\Omega_{1}^{-}\left(E^{k}\right)-\Omega_{1}^{-}\left(E^{k-1}\right)\right) \Omega_{2}^{-}\left(E-E_{k}\right) .
$$

The sum is finite, since $\Omega_{2}^{-}\left(E-E^{k}\right)=0$ for $E^{k}>E-E_{20}$.

Now let us choose a $\Delta$ (which may be large compared to $\delta$ ) and collect those terms with energies between $E_{10}+(l-1) \Delta$ and $E_{10}+l \Delta$ :

with

$$
\Omega^{-}(E)=\sum_{l} \sigma_{l},
$$

$$
\sigma_{l}=\sum_{\substack{k \\ E_{10}+(l-1) \Delta \leqq E^{k}<E_{10}+l \Delta}}\left(\Omega_{1}^{-}\left(E^{k}\right)-\Omega_{1}^{-}\left(E^{k-1}\right)\right) \Omega_{2}^{-}\left(E-E^{k}\right) .
$$

Taking the largest possible value for the second factor, we get

$$
\begin{aligned}
\sigma_{l} & \leqq\left(\Omega_{1}^{-}\left(E_{10}+l \Delta\right)-\Omega_{1}^{-}\left(E_{10}+(l-1) \Delta\right)\right) \Omega_{2}^{-}\left(E-E_{10}-(l-1) \Delta\right) \\
& =\Omega_{1}{ }^{\Delta}\left(E_{10}+(l-1) \Delta\right) \Omega_{2}^{-}\left(E-\left(E_{10}+(l-1) \Delta\right)\right) .
\end{aligned}
$$

Similarly,

$$
\sigma_{l} \geqq \Omega_{1}{ }^{\Delta}\left(E_{10}+(l-1) \Delta\right) \Omega_{2}^{-}\left(E-\left(E_{10}+l \Delta\right)\right) .
$$

Here we have slightly overestimated the first factor, if $\Delta$ is not an exact multiple of $\delta$; but as $E \rightarrow \infty$ and $\Lambda \rightarrow \infty$ we have to choose smaller and smaller $\delta$ 's, whereas $\Delta$ will be kept fixed; so let us assume that $\Delta$ is a multiple of $\delta$. The above estimates imply

$$
\begin{aligned}
\sum_{l} \Omega_{1}{ }^{\Delta}\left(E_{10}+(l-1) \Delta\right) \Omega_{2}^{-}\left(E-\left(E_{10}+(l-1) \Delta\right)\right) \geqq \Omega^{-}(E) \\
\quad \geqq \sum_{l} \Omega_{1}{ }^{\Delta}\left(E_{10}+(l-1) \Delta\right) \Omega_{2}^{-}\left(E-\Delta-\left(E_{10}+(l-1) \Delta\right)\right) \\
\quad \geqq \Omega^{-}(E-\Delta) .
\end{aligned}
$$


$\Delta$ is fixed, hence $V(\Lambda)^{-1}(E-\Delta) \rightarrow \varepsilon$ and $\lim V^{-1} \log \Omega^{-}(E)=\lim V^{-1}$ $\cdot \log \Omega^{-}(E-\Delta)$. Therefore we can find a correction factor $\xi(\Lambda)$ such that

with

$$
\Omega^{-}(E)=\xi(\Lambda) \sum_{k} \Omega_{1}{ }^{\Delta}\left(E_{10}+k \Delta\right) \Omega_{2}^{-}\left(E-\left(E_{10}+k \Delta\right)\right)
$$

Denote the right hand side of $(9)$ by $s^{0}(\varrho, \varepsilon)$; we want to prove our theorem by showing that $\liminf _{\Lambda \rightarrow \infty} V(\Lambda)^{-1} \log \Omega^{-}(E) \geqq s^{0}(\varrho, \varepsilon), \quad E=V(\Lambda) \varepsilon$, and $\limsup _{\Lambda \rightarrow \infty} V(\Lambda)^{-1} \log \Omega^{-}(E) \leqq s^{0}(\varrho, \varepsilon)$.

We have to care only for $\varepsilon^{\prime} \in I(\varepsilon)$, since otherwise the arguments of $s_{i}$ are not larger than $\varepsilon_{10}(\varrho)$ and $\varepsilon_{20}(\varrho)$, respectively.

(i) Choose an arbitrary $\delta>0$. There exists an interval $I_{\delta}=\left(\varepsilon_{1}, \varepsilon_{2}\right) \subseteq I(\varepsilon)$ such that for $\varepsilon^{\prime} \in I_{\delta}$

$$
\kappa_{1} s_{1}\left(\varrho, \frac{\varepsilon^{\prime}}{\kappa_{1}}\right)+\kappa_{2} s_{2}\left(\varrho, \frac{\varepsilon-\varepsilon^{\prime}}{\kappa_{2}}\right)>s^{0}(\varrho, \varepsilon)-\delta .
$$

We approximate $s_{1}\left(\varrho, \frac{\varepsilon^{\prime}}{\kappa_{1}}\right)$ by $V(\Lambda)^{-1} \log \Omega_{1}{ }^{\Delta}\left(\Lambda_{1}, n_{1}, E_{1}^{\prime}\right)$ and $s_{2}\left(\varrho, \frac{\varepsilon-\varepsilon^{\prime}}{\kappa_{2}}\right)$ by $V\left(\Lambda_{2}\right)^{-1} \log \Omega_{2}^{-}\left(\Lambda_{2}, n_{2}, E_{2}^{\prime}\right)$, with

$$
\begin{aligned}
& E_{1}^{\prime}=V\left(\Lambda_{1}\right) \frac{\varepsilon^{\prime}}{\kappa_{1}}=V(\Lambda) \varepsilon^{\prime}, \\
& E_{2}^{\prime}=V\left(\Lambda_{2}\right) \frac{\varepsilon-\varepsilon^{\prime}}{\kappa_{2}}=V(\Lambda)\left(\varepsilon-\varepsilon^{\prime}\right),
\end{aligned}
$$

[where we made use of (6)], and get

$$
\begin{gathered}
\kappa_{1} V\left(\Lambda_{1}\right)^{-1} \log \Omega_{1}{ }^{\Delta}\left(E_{1}^{\prime}\right)+\kappa_{2} V\left(\Lambda_{2}\right)^{-1} \log \Omega_{2}^{-}\left(E_{2}^{\prime}\right) \\
=V(\Lambda)^{-1}\left(\log \Omega_{1}{ }^{\Delta}\left(E_{1}^{\prime}\right)+\log \Omega_{2}^{-}\left(E_{2}^{\prime}\right)\right) \\
\quad>s^{0}(\varrho, \varepsilon)-2 \delta
\end{gathered}
$$

for sufficiently large $\Lambda$. (The $s_{i}$ are continuous functions, thus $\varepsilon^{\prime}$ varies over a compact set, and the convergence of $V^{-1} \log \Omega^{-}$is uniform on compact sets [2].) Put $E=V(\Lambda) \varepsilon$ in (11) and adjust $\varepsilon^{\prime} \in I_{\delta}$ such that $V(\Lambda) \varepsilon^{\prime}=E_{10}+k \Delta$ for a suitable $k$ (which is always possible if $\Lambda$ is large enough), then

$$
\begin{aligned}
\xi(\Lambda)^{-1} \Omega^{-}(V(\Lambda) \varepsilon) \geqq & \Omega_{1}{ }^{A}\left(V(\Lambda) \varepsilon^{\prime}\right) \Omega_{2}^{-}\left(V(\Lambda)\left(\varepsilon-\varepsilon^{\prime}\right)\right) \\
& >\exp V(\Lambda)\left(s^{0}(\varrho, \varepsilon)-2 \delta\right),
\end{aligned}
$$

and therefore

$$
\begin{aligned}
& \liminf _{\Lambda \rightarrow \infty} V(\Lambda)^{-1}\left(\log \Omega^{-}(V(\Lambda) \varepsilon)-\log \xi(\Lambda)\right) \\
& \quad=\liminf _{\Lambda \rightarrow \infty} V(\Lambda)^{-1} \log \Omega^{-}(V(\Lambda) \varepsilon) \geqq s^{0}(\varrho, \varepsilon) .
\end{aligned}
$$


(ii) For $\varepsilon^{\prime} \in I(\varepsilon)$ and for arbitrary $\delta>0$, we have for sufficiently large $\Lambda$

$$
s^{0}(\varrho, \varepsilon)+\delta \geqq V(\Lambda)^{-1}\left(\log \Omega_{1}{ }^{4}\left(V(\Lambda) \varepsilon^{\prime}\right)+\log \Omega_{2}^{-}\left(V(\Lambda)\left(\varepsilon-\varepsilon^{\prime}\right)\right)\right) .
$$

The summation in (11) extends from $k=0$ to some $k \leqq \Delta^{-1}\left(E-E_{10}-E_{20}\right)$; let the largest term of the sum be given by $k_{0}$, then

$\Omega^{-}(E) \leqq \xi(\Lambda) \frac{E-E_{10}-E_{20}}{\Delta} \Omega_{1}{ }^{\Delta}\left(E_{10}+k_{0} \Delta\right) \Omega_{2}^{-}\left(E-\left(E_{10}+k_{0} \Delta\right)\right)$.

Combining (13) and (14), with $\varepsilon^{\prime}=V(\Lambda)^{-1}\left(E_{10}+k_{0} \Delta\right)$, we conclude

$$
\begin{aligned}
s^{0}(\varrho, \varepsilon)+\delta & \geqq V(\Lambda)^{-1} \log \Omega_{1}{ }^{\Delta}\left(E_{10}+k_{0} \Delta\right) \Omega_{2}^{-}\left(V(\Lambda) \varepsilon-\left(E_{10}+k_{0} \Delta\right)\right) \\
& \geqq V(\Lambda)^{-1} \log \left(\xi(\Lambda) \Delta^{-1}\left(V(\Lambda) \varepsilon-E_{10}-E_{20}\right)\right)^{-1} \Omega^{-}(V(\Lambda) \varepsilon),
\end{aligned}
$$

and since $V(\Lambda)^{-1} \log \xi(\Lambda) \rightarrow 0, V(\Lambda)^{-1}\left(\log \Lambda+\log \left(V(\Lambda) \varepsilon-E_{10}-E_{20}\right)\right) \rightarrow 0$, we arrive at

$$
s^{0}(\varrho, \varepsilon) \geqq \limsup _{\Lambda \rightarrow \infty} V(\Lambda)^{-1} \log \Omega^{-}(E) .
$$

(12) and (15) together with Proposition 2.1 prove our theorem.

Remark 2.4. The ground state energy $E_{0}(\Lambda, n)$ of $H_{0}(\Lambda, n)$ is given by $E_{10}\left(\Lambda_{1}, n_{1}\right)+E_{20}\left(\Lambda_{2}, n_{2}\right)$, thence

$$
\lim V(\Lambda)^{-1} E_{0}(\Lambda, n)=\varepsilon_{0}(\varrho)=\kappa_{1} \varepsilon_{10}(\varrho)+\kappa_{2} \varepsilon_{20}(\varrho) .
$$

We are interested in the case $\varepsilon>\varepsilon_{0}(\varrho)$, and this implies that $I(\varepsilon)$ is not empty.

Remark 2.5. $I(\varepsilon)$ is a closed interval, thus there exists an $\varepsilon_{m}$ for which the supremum in (9) is achieved:

$$
\lim _{\lambda \rightarrow 0} s_{\lambda}(\varrho, \varepsilon)=s_{0}(\varrho, \varepsilon)=\kappa_{1} s_{1}\left(\varrho,-\frac{\varepsilon_{m}}{\kappa_{1}}\right)+\kappa_{2} s_{2}\left(\varrho, \frac{\varepsilon-\varepsilon_{m}}{\kappa_{2}}\right) .
$$

Remembering that $s_{\lambda}, S_{l}, \varepsilon$, and $\varepsilon_{m}$ are densities, one realizes that the additivity of the entropy is adequately expressed by (16).

Remark 2.6. The condition $\Lambda_{1} \cap \Lambda_{2}=\emptyset$ was used in order to avoid mixing entropy: the essential point is that we wanted to use the Hilbert spaces $\mathscr{H}(\Lambda)=\mathscr{H}_{1}\left(\Lambda_{1}\right) \otimes \mathscr{H}_{2}\left(\Lambda_{2}\right)$ without additional symmetrization. $\mathscr{H}_{1}$ and $\mathscr{H}_{2}$, of course, have to contain appropriately symmetrized wave functions. The non-symmetrization does in no respect affect Ruelle's proof of the existence of $s(\varrho, \varepsilon)$. The same holds true, if we consider different sorts of particles in $\mathscr{S}_{1}$ and $\mathscr{S}_{2}$, respectively. In that case, we can distinguish between them without enclosing the systems in nonoverlapping regions of space, and can hence drop the above condition. 
We might, for instance, consider a system $\mathscr{S}_{1}$ consisting of nuclei with a weak spin coupling to an electron gas representing $\mathscr{S}_{2}$. We may choose different densities $\varrho_{1}$ and $\varrho_{2}$ as well. In that case, one has to adapt Ruelle's proof in order to show the existence of

$$
\lim _{\Lambda \rightarrow \infty} V(\Lambda)^{-1} \log \Omega_{\lambda}\left(\Lambda, n_{1}, n_{2}, E\right)=s_{\lambda}\left(\varrho_{1}, \varrho_{2}, \varepsilon\right) .
$$

The theorem remains valid:

$$
\begin{aligned}
\lim _{\lambda \rightarrow \infty} s_{\lambda}\left(\varrho_{1}, \varrho_{2}, \varepsilon\right) & =\sup _{\varepsilon^{\prime} \in I(\varepsilon)}\left(\kappa_{1} s_{1}\left(\varrho_{1}, \frac{\varepsilon^{\prime}}{\kappa_{1}}\right)+\kappa_{2} s_{2}\left(\varrho_{2}, \frac{\varepsilon-\varepsilon^{\prime}}{\kappa_{2}}\right)\right), \\
I(\varepsilon) & =\left[\kappa_{1} \varepsilon_{10}\left(\varrho_{1}\right), \varepsilon-\kappa_{2} \varepsilon_{20}\left(\varrho_{2}\right)\right] .
\end{aligned}
$$

\section{Discussion of the Additivity Equation, Properties of $s(\varrho, \varepsilon)$}

This section is devoted to a short discussion of the additivity Eq. (9) which leads - at least in some cases - to the definition of the temperature as derivative of $s$, and to a summary of properties of the entropy densities needed in the next section. In this section and in the following one, we shall always suppress the arguments $\varrho$ and $\varrho_{i}$; the densities will be kept fixed.

Remark 3.1. It is well known that the entropy densities are continuous, concave, increasing functions of the respective energy densities [2]. This implies

(i) their left and right derivatives $s_{-}^{\prime}, s_{+}^{\prime}$ exist everywhere;

(ii) $s_{+}^{\prime}(\varepsilon) \leqq S_{-}^{\prime}(\varepsilon)$;

(iii) left and right derivatives are equal almost everywhere.

For the proof see for instance [3]. (The essential point is the concavity of $s$.)

(iv) Moreover, because $s$ is increasing, the number of exceptional points where $s_{+}^{\prime} \neq s_{-}^{\prime}$ is at most countable.

Remark 3.2. Define

$$
g_{\kappa_{1}}\left(\varepsilon^{\prime}\right)=\kappa_{1} s_{1}\left(\frac{\varepsilon^{\prime}}{\kappa_{1}}\right)+\kappa_{2} s_{2}\left(\frac{\varepsilon-\varepsilon^{\prime}}{\kappa_{2}}\right),
$$

so that Eq. (9) reads

$$
s(\varepsilon)=\sup _{\varepsilon^{\prime} \in I(\varepsilon)} g_{\kappa_{1}}\left(\varepsilon^{\prime}\right) .
$$

We have to discern three cases:

(i) The supremum is reached at an inner point of $I(\varepsilon)$;

(ii) the supremum is reached for $\varepsilon^{\prime}=\kappa_{1} \varepsilon_{10}$;

(iii) the supremum is reached for $\varepsilon^{\prime}=\varepsilon-\kappa_{2} \varepsilon_{20}$. 
Since the $s_{i}$ are concave functions, $g_{\kappa_{1}}$ is concave too, and can have at most one maximum which in case (i) is given by

$$
\left(g_{\kappa_{1}}\right)_{+}^{\prime}\left(\varepsilon_{m}\right) \leqq 0, \quad\left(g_{\kappa_{1}}\right)_{-}^{\prime}\left(\varepsilon_{m}\right) \geqq 0,
$$

or, equivalently, by

$$
s_{1+}^{\prime}\left(\frac{\varepsilon_{m}}{\kappa_{1}}\right) \leqq s_{2}^{\prime}-\left(\frac{\varepsilon-\varepsilon_{m}}{\kappa_{2}}\right), \quad s_{1}^{\prime}-\left(\frac{\varepsilon_{m}}{\kappa_{1}}\right) \geqq s_{2+}^{\prime}\left(\frac{\varepsilon-\varepsilon_{m}}{\kappa_{2}}\right) .
$$

$\left[\right.$ Here $s_{1}^{\prime}+\left(\frac{\varepsilon_{m}}{\kappa_{1}}\right)$ means $\left.s_{1}^{\prime}\left(\varepsilon^{\prime}\right)\right|_{\varepsilon^{\prime}=\varepsilon_{m} / \kappa_{1}}$, etc. $]$.

If the left and right derivatives of $s_{1}$ and $s_{2}$ at the respective points are equal, (18a) and (18b) go over into

$$
s_{1}^{\prime}\left(\frac{\varepsilon_{m}}{\kappa_{1}}\right)=s_{2}^{\prime}\left(\frac{\varepsilon-\varepsilon_{m}}{\kappa_{2}}\right) .
$$

In this case, we can define a unique inverse equilibrium temperature by

$$
\beta=s_{1}^{\prime}\left(\frac{\varepsilon_{m}}{\kappa_{1}}\right) \text {. }
$$

The maximum of $g$ may be a plateau. Eqs. $(18 \mathrm{a}, \mathrm{b})$ then fail to determine $\varepsilon_{m}$ uniquely. We shall later remove this ambiguity by taking the largest or the smallest solutions of $(18 \mathrm{a}, \mathrm{b})$.

It is easily seen that corresponding equations hold also for three or more systems in equilibrium with each other.

In case (ii), we have only Eq. (18a):

$$
s_{1+}^{\prime}\left(\varepsilon_{10}\right) \leqq s_{2}^{\prime}-\left(\frac{\varepsilon-\kappa_{1} \varepsilon_{10}}{\kappa_{2}}\right)
$$

This means that the system $\mathscr{S}_{1}$ is in its ground state even for temperatures larger than zero. Whenever such a system exists, the notion of temperature is meaningless for it in this state. Therefore, we shall exclude this case. Case (iii) can also easily be excluded, if we consider $\kappa_{1} \rightarrow 0$ and if $\varepsilon>\varepsilon_{0}$. We would then get

$$
s_{1}^{\prime}-\left(\frac{\varepsilon-\kappa_{2} \varepsilon_{20}}{\kappa_{1}}\right) \geqq s_{2+}^{\prime}\left(\varepsilon_{20}\right) .
$$

But as $\kappa_{1} \rightarrow 0$ the argument of $s_{1}^{\prime}$ - increases, and we know [2] that the lower limit of the slopes of the tengents on the graph $\left(\varepsilon, s_{1}(\varepsilon)\right)$ is zero, hence $s_{1}$ - must finally become smaller than $s_{2}{ }^{\prime}\left(\varepsilon_{20}\right)$, thus violating the above equation. [If $\varepsilon=\varepsilon_{0}$, then $\frac{\varepsilon-\kappa_{2} \varepsilon_{20}}{\kappa_{1}}=\frac{\kappa_{1} \varepsilon_{10}}{\kappa_{1}}$ would not increase.] 
From now on, we are interested in a system in contact with a heat reservoir which has to be infinitely large compared to the system $\mathscr{S}_{1}$ considered. The relative size of $\mathscr{S}_{1}$ is given by $\kappa_{1}$ therefore, we shall consider the limit $\kappa_{1} \rightarrow 0$.

If we perform this limit, the temperature $1 / \beta$ turns out to be uniquely defined (apart from a special case), even if the left and right derivatives of $s_{1}$ and $s_{2}$ may differ at many points: $\beta=s_{2}^{\prime}(\varepsilon)$ or $\beta=s_{2}^{\prime}(\varepsilon)$, depending on the functional shape of $s_{1}$ and $s_{2}$.

For the next section, we need some information on the behaviour of $\varepsilon_{m}\left(\kappa_{1}\right)$ if $\kappa_{1} \rightarrow 0$. $\varepsilon$ is henceforward considered as a fixed parameter.

Lemma 3.3. There exists an $\varepsilon_{1}$ such that

hence

$$
\kappa_{1} \varepsilon_{10} \leqq \varepsilon_{m}\left(\kappa_{1}\right)<\kappa_{1} \varepsilon_{1}
$$

$$
\lim _{\kappa_{1} \rightarrow 0} \varepsilon_{m}\left(\kappa_{1}\right)=0, \quad \lim _{\kappa_{1} \rightarrow 0} \frac{\varepsilon-\varepsilon_{m}\left(\kappa_{1}\right)}{\kappa_{2}}=\varepsilon
$$

Proof. As already mentioned, the lower limit of the slopes of the tangents is zero, hence

$$
s_{1}^{\prime}-\left(\varepsilon_{1}\right)<s_{2}^{\prime}-(\varepsilon)
$$

for a sufficiently large $\varepsilon_{1}$. We may assume $\varepsilon_{1}>\varepsilon$, and thus

$$
\frac{\varepsilon-\kappa_{1} \varepsilon}{\kappa_{2}}=\varepsilon-\frac{\kappa_{1}}{\kappa_{2}}\left(\varepsilon_{1}-\varepsilon\right)<\varepsilon
$$

This implies

$$
S_{2+}^{\prime}\left(\frac{\varepsilon-\kappa_{1} \varepsilon}{\kappa_{2}}\right) \geqq S_{2-}^{\prime}(\varepsilon)
$$

(see Appendix B). According to (20), we can find a $\delta>0$ with $s_{1}^{\prime}\left(\varepsilon_{1}\right)$ $<s_{2}^{\prime}-(\varepsilon)-\delta$. But then

$$
\left(g_{\kappa_{1}}\right)_{-}^{\prime}\left(\kappa_{1} \varepsilon_{1}\right)=s_{1}^{\prime}\left(\varepsilon_{1}\right)-s_{2+}^{\prime}\left(\frac{\varepsilon-\kappa_{1} \varepsilon_{1}}{\kappa_{2}}\right)<0 .
$$

Thus the maximum condition $\left(g_{\kappa_{1}}\right)_{-}^{\prime}\left(\varepsilon_{m}\right) \geqq 0$ can only be fulfilled if $\varepsilon_{m}<\kappa_{1} \varepsilon_{1}$ (notice that $g_{\kappa_{1}}$ is concave). The lower limit for $\varepsilon_{m}$ is trivial because $\varepsilon_{m}\left(\kappa_{1}\right) \in I(\varepsilon)$.

Q.E.D.

Let us define

$$
\eta\left(\kappa_{1}\right)=\frac{\varepsilon_{m}\left(\kappa_{1}\right)}{\kappa_{1}}, \quad \tilde{\varepsilon}\left(\kappa_{1}\right)=\varepsilon-\frac{\kappa_{1}}{\kappa_{2}}\left(\eta\left(\kappa_{1}\right)-\varepsilon\right)=\frac{\varepsilon-\varepsilon_{m}\left(\kappa_{1}\right)}{\kappa_{2}} .
$$

Then we can rewrite the supremum conditions:

$$
s_{1+}^{\prime}\left(\eta\left(\kappa_{1}\right)\right) \leqq s_{2}^{\prime}\left(\tilde{\varepsilon}\left(\kappa_{1}\right)\right), \quad s_{1-}^{\prime}\left(\eta\left(\kappa_{1}\right)\right) \geqq s_{2+}^{\prime}\left(\tilde{\varepsilon}\left(\kappa_{1}\right)\right) . \quad(22 \mathrm{a}, \mathrm{b})
$$


There are three cases:

Case $(\varepsilon): \quad s_{1}^{\prime}+(\varepsilon) \leqq s_{2}^{\prime}-(\varepsilon), s_{1}^{\prime}-(\varepsilon) \geqq s_{2}^{\prime}+(\varepsilon)$;

Case $\left(\varepsilon_{1}\right)$ : the first of these inequalities is violated:

$$
s_{1}^{\prime}+(\varepsilon)>s_{2-}^{\prime}(\varepsilon) ;
$$

Case $\left(\varepsilon_{2}\right)$ : the second inequality is violated:

$$
s_{1}^{\prime}-(\varepsilon)<s_{2}^{\prime}(\varepsilon) .
$$

We can add two further inequalities to (23) [see Remark 3.1 (ii)]:

$$
s_{1}^{\prime}(\varepsilon) \geqq s_{1+}^{\prime}(\varepsilon)>s_{2}^{\prime}-(\varepsilon) \geqq s_{2+}^{\prime}(\varepsilon),
$$

thus showing that the second inequality of case $(\varepsilon)$ is fulfilled. Similarly, in case $\left(\varepsilon_{2}\right)$ the first inequality is valid; hence all possibilities are exhausted.

Case $(\varepsilon)$ implies that we can choose $\eta\left(\kappa_{1}\right)=\varepsilon$, hence $\tilde{\varepsilon}\left(\kappa_{1}\right)=\varepsilon$, for all $\kappa_{1}$. This means that $\mathscr{S}_{1}$ and $\mathscr{S}_{2}$, when in equilibrium with each other, always have the same energy densities, irrespective of their relative "size" $\kappa_{1} / \kappa_{2}$

Lemma 3.4. Given case $\left(\varepsilon_{1}\right)$ (resp. case $\left(\varepsilon_{2}\right)$ ), then

(i) $\eta\left(\kappa_{1}\right)>\varepsilon\left(\right.$ resp. $\left.\eta\left(\kappa_{1}\right)<\varepsilon\right)$ for all $\kappa_{1}$;

(ii) $s_{2 \pm}^{\prime}\left(\tilde{\varepsilon}\left(\kappa_{1}\right)\right) \underset{\kappa_{1} \rightarrow 0}{\longrightarrow} s_{2}^{\prime}-(\varepsilon)$ (resp. $\left.s_{2}^{\prime}(\varepsilon)\right)$.

This result already indicates that $s_{2}{ }^{\prime}(\varepsilon)$ or $s_{2}{ }_{-}^{\prime}(\varepsilon)$ will be the temperature defining quantities in the limit $\kappa_{1} \rightarrow 0$.

Proof. We consider case $\left(\varepsilon_{1}\right)$, case $\left(\varepsilon_{2}\right)$ can be treated completely analogously.

(i) Suppose there exists a $\kappa_{1}$ with

then

$$
\eta\left(\kappa_{1}\right) \leqq \varepsilon
$$

and hence

$$
\tilde{\varepsilon}\left(\kappa_{1}\right)=\varepsilon-\frac{\kappa_{1}}{\kappa_{2}}\left(\eta\left(\kappa_{1}\right)-\varepsilon\right) \geqq \varepsilon
$$

$$
s_{1}^{\prime}\left(\eta\left(\kappa_{1}\right)\right) \geqq s_{1}^{\prime}(\varepsilon)>s_{2}^{\prime}-(\varepsilon) \geqq s_{2}^{\prime}-\left(\widetilde{\varepsilon}\left(\kappa_{1}\right)\right),
$$

in contradiction to $(22 \mathrm{a})$. [The first and the last inequalities are due to (25) and (26), the second one due to (23); cf. Appendix B.]

(ii) Clearly, $\eta\left(\kappa_{1}\right)>\varepsilon$ implies $\tilde{\varepsilon}\left(\kappa_{1}\right)<\varepsilon$. According to Lemma 3.3, $\tilde{\varepsilon}\left(\kappa_{1}\right) \underset{\kappa_{1} \rightarrow 0}{\longrightarrow} \varepsilon$ and thence $s_{2 \pm}^{\prime}\left(\tilde{\varepsilon}\left(\kappa_{1}\right)\right) \underset{\kappa_{1} \rightarrow 0}{\longrightarrow} s_{2-}^{\prime}(\varepsilon)$, (see also Appendix B).

Up to now we need not assume that $\eta\left(\kappa_{1}\right)$ is uniquely defined by $(22 \mathrm{a}, \mathrm{b})$. In order to get uniqueness, let us define

$$
\begin{aligned}
& \eta_{s}\left(\kappa_{1}\right)=\max \left\{\eta\left(\kappa_{1}\right) ; \kappa_{1} \text { fixed, } \eta\left(\kappa_{1}\right) \text { solution of }(22 \mathrm{a}, \mathrm{b})\right\}, \\
& \eta_{i}\left(\kappa_{1}\right)=\min \left\{\eta\left(\kappa_{1}\right) ; \kappa_{1} \text { fixed, } \eta\left(\kappa_{1}\right) \text { solution of }(22 \mathrm{a}, \mathrm{b})\right\} .
\end{aligned}
$$


Min and max of the above set $\{\ldots\}$ exist because $g_{\kappa_{1}}$ is continuous. Accordingly, we write

$$
\tilde{\varepsilon}_{s}\left(\kappa_{1}\right)=\varepsilon-\frac{\kappa_{1}}{\kappa_{2}}\left(\eta_{s}-\varepsilon\right) \text { and } \tilde{\varepsilon}_{i}\left(\kappa_{1}\right)=\varepsilon-\frac{\kappa_{1}}{\kappa_{2}}\left(\eta_{i}-\varepsilon\right) .
$$

Lemma 3.5. (i) Case $\left(\varepsilon_{1}\right): \eta_{s}\left(\kappa_{1}\right)$ increases with decreasing $\kappa_{1}$, case $\left(\varepsilon_{2}\right): \eta_{i}\left(\kappa_{1}\right)$ decreases with decreasing $\kappa_{1}$.

(ii) Define

$$
K=\left\{\kappa_{1} ; s_{2+}^{\prime}\left(\tilde{\varepsilon}\left(\kappa_{1}\right)\right)=s_{2}^{\prime}\left(\tilde{\varepsilon}\left(\kappa_{1}\right)\right)\right\},
$$

where $\tilde{\varepsilon}$ means $\tilde{\varepsilon}_{\mathrm{s}}$ in case $\left(\varepsilon_{1}\right)$ and $\tilde{\varepsilon}_{i}$ in case $\left(\varepsilon_{2}\right)$. In both cases, $K$ contains sequences $\kappa_{1 v} \rightarrow 0$.

The proof will be given in Appendix C.

Part (i) together with Lemma 3.3 implies that $\lim _{\kappa_{1} \rightarrow 0} \eta\left(\kappa_{1}\right)$ exists, i.e. the energy density of $\mathscr{S}_{1}$ is well defined for $\kappa_{1} \rightarrow 0$, as is to be expected if the limiting procedure makes sense.

\section{Systems in Contact with a Heat Reservoir and Transition to Canonical Ensemble States}

In this section, we are interested in a system $\mathscr{S}_{1}$ in thermal equilibrium with a heat reservoir, i.e. with a system $\mathscr{S}_{2}$ which is large compared to $\mathscr{S}_{1}$; the systems are the same as in Section II, but now we take, in addition, the limit $\kappa_{1} \rightarrow 0$.

The basic assumption of this section is the following:

$\left(\mathrm{A}_{2}\right)$ The equilibrium states of a closed system which is described by a microcanonical ensemble are given by

$$
\begin{aligned}
\omega_{\varepsilon}(A) & =\lim _{\Lambda \rightarrow \infty} \omega_{\Lambda}{ }^{E}(A), \\
\omega_{\Lambda}{ }^{E}(A) & =\frac{\operatorname{Tr} \delta^{\Lambda}(H(\Lambda)-E) A}{\operatorname{Tr} \delta^{\Lambda}(H(\Lambda)-E)},
\end{aligned}
$$

where $A$ is an element of a suitably chosen observable algebra $\mathfrak{A}$, and $\delta^{A}(x)$ is the function defined after $\left(\mathrm{A}_{1}\right)$ in Section II.

Part of this axiom is a further specification of axiom $\left(\mathrm{A}_{0}\right)$ of the introduction, part of it is an additional assumption: the existence of the above limit. We are not going to answer the question under what conditions this limit exists. Consequently, we cannot show, that the limits $\lambda \rightarrow 0$ and $\Lambda \rightarrow \infty$ can be interchanged for the states, as they can for the entropy density (Proposition 2.1). We shall therefore put $\lambda=0$ from the beginning, and start with Hamiltonians $H(\Lambda)=H_{1}\left(\Lambda_{1}\right)+H_{2}\left(\Lambda_{2}\right)$, operating in Hilbert spaces $\mathscr{H}(\Lambda)=\mathscr{H}_{1}\left(\Lambda_{1}\right) \otimes \mathscr{H}_{2}\left(\Lambda_{2}\right)$. To $\mathscr{S}_{1}, \mathscr{S}_{2}$, and $\mathscr{S}$ we 
ascribe observable algebras $\mathfrak{A}_{1}, \mathfrak{A}_{2}, \mathfrak{A}$ with some local structure. We need not make detailed assumptions; the essential point is that we can embed $\mathfrak{U}_{1}$ into $\mathfrak{U}$ in the form $\mathfrak{A}_{1} \otimes 1 . \omega_{\varepsilon}$ restricted to $\mathfrak{U}_{1} \otimes \mathbf{1}$ can be considered as a state over $\mathfrak{U}_{1}$. Our aim is to show that, in the limit $\kappa_{1} \rightarrow 0$, this state is a "canonical" one, more precisely: to establish the following theorem:

Theorem 4.1. Let $\left(\mathrm{A}_{2}\right)$ be fulfilled for all $\kappa_{1}$, more precisely, we assume the following: $\lim _{\Lambda_{1}, \Lambda_{2} \rightarrow \infty} \omega_{\Lambda_{1} \cup \Lambda_{2}} E(A)$ exists, in whatever way the limits $\Lambda_{1} \rightarrow \infty$ and $\Lambda_{2} \rightarrow \infty$ are coupled, provided they are accomplished in the sense of Fisher. If the limiting procedures are classified according to their respective values $\kappa_{1}=\lim _{\Lambda_{1}, \Lambda_{2} \rightarrow 0} V\left(\Lambda_{1}\right)\left(V\left(\Lambda_{1}\right)+V\left(\Lambda_{2}\right)\right)^{-1}$, then limiting procedures of the same class yield the same state $\omega_{\varepsilon}{ }^{\kappa_{1}}$. Furthermore, assume whatever is suitable to ensure the result of Theorem 2.3.

If $\varepsilon>\max \left(\varepsilon_{10}, \varepsilon_{20}\right)$, then exists a sequence $\xi_{\Lambda_{1}}$ of real positive numbers with $\lim _{\Lambda_{1} \rightarrow \infty} \xi_{\Lambda_{1}}=\beta$, and

$\beta$ is given by

$$
\omega_{\varepsilon}{ }^{\kappa_{1}=0}(A \otimes \mathbf{1})=\lim _{\Lambda_{1} \rightarrow \infty} \frac{\operatorname{Tr} e^{-\xi_{\Lambda_{1}} H_{1}\left(\Lambda_{1}\right)} A}{\operatorname{Tr} e^{-\xi_{\Lambda_{1}} H_{1}\left(\Lambda_{1}\right)}}, \quad A \in \bigcup_{\Lambda_{1}} \mathfrak{A}_{1}\left(\Lambda_{1}\right) .
$$

$$
\beta= \begin{cases}s_{2}^{\prime}-(\varepsilon) & \text { in case }\left(\varepsilon_{1}\right) \\ s_{2}^{\prime}(\varepsilon) & \text { in case }\left(\varepsilon_{2}\right) \\ s_{2}^{\prime}(\varepsilon) & \text { in case }(\varepsilon), \text { if } s_{2}^{\prime}+(\varepsilon)=s_{2}^{\prime}(\varepsilon) .\end{cases}
$$

The different cases are defined as in the preceding section after Eq. (22). The rest of this section is devoted to the proof of this theorem. Due to the condition $\varepsilon>\max \left(\varepsilon_{10}, \varepsilon_{20}\right)$, we can exclude the cases $\varepsilon_{m}\left(\kappa_{1}\right)=\kappa_{1} \varepsilon_{10}$ and $\varepsilon_{m}\left(\kappa_{1}\right)=\varepsilon-\kappa_{2} \varepsilon_{20}$, and can apply the results of the preceding section.

By $\psi_{i, r}, i=1,2, \ldots, r=1,2,3, \ldots$, we denote the normalized eigenfunctions of $H_{i}\left(\Lambda_{i}\right)$ with the respective eigenvalues $E_{i, r}$. (We omit further indices $\Lambda_{i}$ indicating the $\Lambda_{i}$-dependence of $\psi_{i, r}$ and $E_{i, r}$. ) Then we can write

$$
\begin{aligned}
\omega_{\Lambda}{ }^{E}(A \otimes \mathbf{1}) & =\left(\Omega^{\Delta}(\Lambda, E)\right)^{-1} \sum_{\substack{r, s \\
E \leqq E_{1 r}+E_{2 s} \leqq E+\Delta}}\left(\psi_{1 r}, A \psi_{1 r}\right)\left(\psi_{2 s}, \psi_{2 s}\right) \\
& =\left(\Omega^{\Delta}(\Lambda, E)\right)^{-1} \sum_{r} \Omega_{2}{ }^{\Delta}\left(\Lambda_{2}, E-E_{1 r}\right)\left(\psi_{1 r}, A \psi_{1 r}\right) .
\end{aligned}
$$

From now on, we also omit the arguments $\Lambda, \Lambda_{2}$ of $\Omega, \Omega_{2}$, and the index $\Delta$. Our task is to give an estimate for $\Omega_{2}\left(E-E_{1 r}\right)$ in terms of const $\cdot e^{-\xi E_{1 r}}$ with an appropriate $\xi$. In order to do so, we first look for an estimate for $s_{2}\left(\varepsilon_{2}\right)$.

We treat the cases $\left(\varepsilon_{1}\right),\left(\varepsilon_{2}\right),(\varepsilon)$ with $s_{2}{ }^{\prime}(\varepsilon)=s_{2+}{ }^{\prime}(\varepsilon)$ simultaneously; accordingly, $\tilde{\varepsilon}\left(\kappa_{1}\right)$ means $\tilde{\varepsilon}_{s}\left(\kappa_{1}\right), \tilde{\varepsilon}_{i}\left(\kappa_{1}\right)$ or $\tilde{\varepsilon}\left(\kappa_{1}\right)=\varepsilon$, respectively. 
Now define

$$
\xi_{ \pm}\left(\kappa_{1}\right)=s_{2 \pm}^{\prime}\left(\tilde{\varepsilon}\left(\kappa_{1}\right)\right) .
$$

In the following, we restrict ourselves to those $\kappa_{1}$ for which $\xi_{+}\left(\kappa_{1}\right)$ $=\xi_{-}\left(\kappa_{1}\right) \equiv \xi\left(\kappa_{1}\right)$.

For the cases $\left(\varepsilon_{1}\right)$ and $\left(\varepsilon_{2}\right)$, Lemma 3.5 ensures that the set $K$ of these $\kappa_{1}$ contains sequences $\kappa_{1 v} \rightarrow 0$ so that we can indeed adopt this restriction. In case $(\varepsilon)$, this is trivial as long as $s_{2}{ }^{\prime}(\varepsilon)=s_{2}{ }^{\prime}(\varepsilon)$.

Because $s_{2}\left(\varepsilon_{2}\right)$ is concave, the tangents

$$
t\left(\varepsilon_{2} ; \kappa_{1}\right)=s_{2}\left(\tilde{\varepsilon}\left(\kappa_{1}\right)\right)+\xi\left(\kappa_{1}\right)\left(\varepsilon_{2}-\tilde{\varepsilon}\left(\kappa_{1}\right)\right)
$$

are always above $s_{2}\left(\varepsilon_{2}\right)$. Now let us choose a $\delta>0$ and define

$$
I_{\delta, \kappa_{1}}=\left\{\varepsilon_{2} ; t\left(\varepsilon_{2} ; \kappa_{1}\right)-\delta<s_{2}\left(\varepsilon_{2}\right)\right\} .
$$

This set is a non-empty interval. Hence we get with $\varepsilon_{2}=\frac{\varepsilon-\varepsilon^{\prime}}{\kappa_{2}}$

$$
\begin{aligned}
s_{2}\left(\tilde{\varepsilon}\left(\kappa_{1}\right)\right) & -\frac{\xi\left(\kappa_{1}\right)}{\kappa_{2}}\left(\varepsilon^{\prime}-\varepsilon_{m}\left(\kappa_{1}\right)\right)-\delta \\
& \leqq s_{2}\left(\frac{\varepsilon-\varepsilon^{\prime}}{\kappa_{2}}\right) \leqq s_{2}\left(\tilde{\varepsilon}\left(\kappa_{1}\right)\right)-\frac{\xi\left(\kappa_{1}\right)}{\kappa_{2}}\left(\varepsilon^{\prime}-\varepsilon_{m}\right),
\end{aligned}
$$

the first inequality being valid for $\frac{\varepsilon-\varepsilon^{\prime}}{\kappa_{2}} \in I_{\delta, \kappa_{1}}$.

Remember that $\lim _{\Lambda_{2} \rightarrow \infty} \delta_{2}\left(\Lambda_{2}, V\left(\Lambda_{2}\right) \varepsilon^{\prime}\right)=0$, where

$$
\delta_{2}\left(\Lambda_{2}, V\left(\Lambda_{2}\right) \varepsilon_{2}\right)=s_{2}\left(\varepsilon_{2}\right)-V\left(\Lambda_{2}\right)^{-1} \log \Omega_{2}\left(\Lambda_{2}, V\left(\Lambda_{2}\right) \varepsilon_{2}\right) .
$$

Multiplying (31) by $V\left(\Lambda_{2}\right)=\kappa_{2} V(\Lambda)$, and putting $E=V(\Lambda) \varepsilon, E_{m}=V(\Lambda) \varepsilon_{m}$, $\varepsilon^{\prime}=V(\Lambda)^{-1} E_{1 r}$, we find

$$
\begin{aligned}
\Omega_{2}(E & \left.-E_{m}\right) e^{\xi E_{m}} e^{\xi E_{1 r}} e^{V\left(\Lambda_{2}\right)\left[\delta_{2}\left(\Lambda_{2}, E-E_{m}\right)-\delta_{2}\left(\Lambda_{2}, E-E_{1 r}\right)\right]} e^{-V(\Lambda) \delta} \\
& \leqq \Omega_{2}\left(E-E_{1 r}\right) \\
& \leqq \Omega_{2}\left(E-E_{m}\right) e^{\xi E_{m}} e^{-\xi E_{1 r}} e^{V\left(\Lambda_{2}\right)\left[\delta_{2}\left(\Lambda_{2}, E-E_{m}\right)-\delta_{2}\left(\Lambda_{2}, E-E_{1 r}\right)\right]} ;
\end{aligned}
$$

with the abbreviations

$$
\begin{aligned}
C_{\Lambda_{2}} & =e^{\xi\left(\kappa_{1}\right) E_{m}} e^{V\left(\Lambda_{2}\right) \delta_{2}\left(\Lambda_{2}, E-E_{m}\right)} \Omega_{2}\left(E-E_{m}\right), \\
d\left(\Lambda_{2}, E_{1 r}\right) & =e^{-V\left(\Lambda_{2}\right) \delta_{2}\left(\Lambda_{2}, E-E_{1 r}\right)}
\end{aligned}
$$

this reads

$$
C_{\Lambda_{2}} e^{-V(\Lambda) \delta} e^{-\xi E_{1 r}} d\left(\Lambda_{2}, E_{1 r}\right) \leqq \Omega_{2}\left(E-E_{1 r}\right) \leqq C_{\Lambda_{2}} e^{-\xi E_{1 r}} d\left(\Lambda_{2}, E_{1 r}\right) .
$$

The first inequality is valid for

$$
E_{1 r} \in \mathscr{I}\left(\delta, \kappa_{1}, \Lambda\right)=\left\{E^{\prime} ; \frac{\varepsilon-V(\Lambda)^{-1} E^{\prime}}{\kappa_{2}} \in I_{\delta, \kappa_{1}}\right\} .
$$


According to Ruelle, $\delta_{2}\left(\Lambda_{2}, V\left(\Lambda_{2}\right) \varepsilon_{2}\right) \rightarrow 0$ uniformly in $\varepsilon_{2}$ on compact subsets of the region of convergence. We are interested in energies $E$ such that $E-E_{1 r} \geqq E_{20}$, since otherwise $\Omega_{2}\left(E-E_{1 r}\right)=0$; furthermore, $E_{1 r} \geqq E_{10}$, hence

$$
\begin{aligned}
\varepsilon_{20}-\delta^{\prime} & \leqq V\left(\Lambda_{2}\right)^{-1}\left(E-E_{1 r}\right) \leqq \frac{1}{\kappa_{2}}\left(\varepsilon+\kappa_{1}\left(\varepsilon_{10}+\delta^{\prime \prime}\right)\right) \\
& \leqq 2\left(\varepsilon+\frac{1}{2}\left(\varepsilon_{10}+\delta^{\prime \prime}\right)\right) \text { for all } \kappa_{1} \leqq \frac{1}{2},
\end{aligned}
$$

$\delta^{\prime}$ and $\delta^{\prime \prime}$ being finite correction terms independent of $\Lambda_{2}$. This is a compact interval, thus we can find a $\Delta\left(\Lambda_{2}\right)$ with

$$
\Delta\left(\Lambda_{2}\right) \geqq\left|\delta_{2}\left(\Lambda_{2}, E-E_{1 r}\right)\right|, \quad \Delta\left(\Lambda_{2}\right) \underset{\Lambda_{2} \rightarrow \infty}{\longrightarrow} 0,
$$

independently of $\kappa_{1}$.

It suffices to consider $\omega_{A}{ }^{E}(A \otimes 1)$ for positive operators $A$. Our assumptions ensure the existence of $\sum_{r} e^{-\xi E_{1 r}}\left(\psi_{1 r}, A \psi_{1 r}\right)$; due to (33),

$$
0 \leqq d\left(\Lambda_{2}, E_{1 r}\right) \leqq e^{V\left(\Lambda_{2}\right) \Delta\left(\Lambda_{2}\right)},
$$

and hence, $\sum_{r} e^{-\xi E_{1 r}}\left(\psi_{1 r}, A \psi_{1 r}\right) d\left(\Lambda_{2}, E_{1 r}\right)$ exists and, because $A \geqq 0$, can be written as

$$
\sum_{r} e^{-\xi E_{1 r}}\left(\psi_{1 r}, A \psi_{1 r}\right) d\left(\Lambda_{2}, E_{1 r}\right)=d_{\Lambda_{2}} \sum_{r} e^{-\xi E_{1 r}}\left(\psi_{1 r}, A \psi_{1 r}\right)
$$

with an appropriate constant $d_{\Lambda_{2}}$.

Now we define

$$
K_{\Lambda}=\Omega(E)^{-1} \operatorname{Tr} e^{-\xi\left(\kappa_{1}\right) H_{1}\left(\Lambda_{1}\right)} C_{\Lambda_{2}} d_{\Lambda_{2}}
$$

$\left(K_{\Lambda}\right.$ is also $\kappa_{1}$-dependent through $\xi$ and $\left.E_{m}\right)$, and

$\omega_{\Lambda_{1}}{ }^{\xi}(A)=\frac{\operatorname{Tr} e^{-\xi H_{1}\left(\Lambda_{1}\right)} A}{\operatorname{Tr} e^{-\xi H_{1}\left(\Lambda_{1}\right)}}=\left(\operatorname{Tr} e^{-\xi H_{1}}\right)^{-1} \sum_{r} e^{-\xi E_{1 r}}\left(\psi_{1 r}, A \psi_{1 r}\right) ;$

combining (28) with (32), (34), and (35) we then get

$$
\begin{gathered}
K_{\Lambda} e^{-V(A) \delta} \omega_{\Lambda_{1}}{ }^{\xi}(A)-R_{\Lambda}(A) \leqq \omega_{\Lambda}{ }^{E}(A \otimes \mathbf{1}) \leqq K_{\Lambda} \omega_{\Lambda_{1}}{ }^{\xi}(A), \\
R_{\Lambda}(A)=\Omega(E)^{-1} \sum_{\text {rest }}\left(C_{\Lambda_{2}} d_{\Lambda_{2}} e^{-V(\Lambda) \delta} e^{-\xi E_{1 r}}-\Omega_{2}\left(E-E_{1 r}\right)\right)\left(\psi_{1 r}, A \psi_{1 r}\right) .
\end{gathered}
$$

$\sum_{\text {rest }}$ means summation over those $r$ 's for which $E_{1 r} \notin \mathscr{I}\left(\delta, \kappa_{1}, \Lambda\right)$. Our aim is now to achieve
(a) $V(\Lambda) \delta \rightarrow 0$,
(b) $R_{\Lambda}(A) \rightarrow 0$,
(c) $K_{\Lambda} \rightarrow 1$

by performing the limits $\delta \rightarrow 0, \Lambda \rightarrow \infty, \kappa_{1} \rightarrow 0$ in a suitable way. 
We need some more definitions:

$$
\begin{aligned}
Q\left(\Lambda_{1}, \xi\right) & =\operatorname{Tr} e^{-\xi H_{1}\left(\Lambda_{1}\right)}=\sum_{r} e^{-\xi E_{1 r}}, \\
Q_{\text {rest }}\left(\Lambda_{1}, \xi\right) & =\sum_{\text {rest }} e^{-\xi E_{1 r}}, \\
Q_{\tilde{E}}\left(\Lambda_{1}, \xi\right) & =\sum_{E_{1 r}>\tilde{E}} e^{-\xi E_{1 r}} .
\end{aligned}
$$

Clearly, for arbitrary $\alpha>0$ there exists $\tilde{E}\left(\Lambda_{1}, \alpha\right)$ such that

$$
Q\left(\Lambda_{1}, \xi\right)^{-1} Q_{\tilde{E}\left(\Lambda_{1}, \alpha\right)}\left(\Lambda_{1}, \xi\right) \leqq \alpha .
$$

We divide the summation interval of $Q\left(\Lambda_{1}, \xi\right)$ into three parts:

$$
\begin{gathered}
\mathscr{I}_{1}: E_{10}\left(\Lambda_{1}\right) \leqq E_{1 r} \leqq E_{m}, \\
\mathscr{I}_{2}: E_{m}<E_{1 r} \leqq \tilde{E}\left(\Lambda_{1}, \alpha\right), \\
\mathscr{I}_{3}: \tilde{E}\left(\Lambda_{1}, \alpha\right)<E_{1 r}<\infty .
\end{gathered}
$$

We want to show that $\mathscr{I}\left(\delta, \kappa_{1}, \Lambda\right)$ increases rapidly enough to cover $\mathscr{I}_{1} \cup \mathscr{I}_{2}$; then follows $Q\left(\Lambda_{1}, \xi\right)^{-1} Q_{\text {rest }}<\alpha$, and, because

we then would have

$$
\begin{aligned}
R_{\Lambda}(A) & \leqq \Omega(E)^{-1} C_{\Lambda_{2}} d_{\Lambda_{2}} e^{-V(\Lambda) \delta} \sum_{\text {rest }} e^{-\xi E_{1 r}}\left(\psi_{1 r}, A \psi_{1 r}\right) \\
& \leqq K_{\Lambda} e^{-V(\Lambda) \delta}\|A\| \frac{Q_{\text {rest }}\left(\Lambda_{1}, \xi\right)}{Q\left(\Lambda_{1}, \xi\right)}
\end{aligned}
$$

$$
R_{\Lambda}(A) \leqq K_{\Lambda} e^{-V(\Lambda) \delta}\|A\| \alpha .
$$

We proceed by estimating the lengths $\ell\left(\mathscr{I}_{1}\right), \ell\left(\mathscr{I}_{2}\right)$ of $\mathscr{I}_{1}$ and $\mathscr{I}_{2}$. We know that

$$
\theta_{1}\left(\Lambda_{1}\right) \equiv V\left(\Lambda_{1}\right)^{-1} E_{10}\left(\Lambda_{1}\right)-\varepsilon_{10} \underset{\Lambda_{1} \rightarrow \infty}{\longrightarrow} 0
$$

hence, using Lemma 3.3, we can find an upper bound of $\ell\left(\mathscr{I}_{1}\right)$ :

$$
\begin{aligned}
\ell\left(\mathscr{I}_{1}\right) & =V(\Lambda) \varepsilon_{m}-V\left(\Lambda_{1}\right)\left(\varepsilon_{10}+\theta_{1}\left(\Lambda_{1}\right)\right) \\
& \leqq V(\Lambda) \kappa_{1} \varepsilon_{1}-\kappa_{1} V(\Lambda)\left(\varepsilon_{10}+\theta_{1}\left(\Lambda_{1}\right)\right) \\
& \leqq \text { const } \kappa_{1} V(\Lambda)
\end{aligned}
$$

A similar estimate can be given for $\ell\left(\mathscr{I}_{2}\right)$ :

Lemma 4.2. Let $E_{1}\left(\Lambda_{1}, S_{1}\right)$ denote the inverse function of $S_{1}\left(\Lambda_{1}, E_{1}\right)$ $=\log \Omega_{1}\left(\Lambda_{1}, E_{1}\right)$. We can choose

$$
\tilde{E}\left(\Lambda_{1}, \alpha\right)=E_{1}\left(\Lambda_{1}, V(\Lambda) \frac{s_{0}}{\alpha}\right)
$$


in order to get

$$
Q\left(\Lambda_{1}, \xi\right)^{-1} Q_{\tilde{E}\left(\Lambda_{1}, \alpha\right)}\left(\Lambda_{1}, \xi\right) \leqq \alpha
$$

for all $\xi$ in a finite interval $\left(\xi_{1}, \xi_{2}\right)$. $s_{0}$ is a constant which depends only on $\left(\xi_{1}, \xi_{2}\right)$.

Proof. We define

$$
\begin{aligned}
f\left(\Lambda_{1}, \xi\right) & =-\xi^{-1} V\left(\Lambda_{1}\right)^{-1} \log Q\left(\Lambda_{1}, \xi\right), \\
s_{\Lambda_{1}}(\xi) & =\xi^{2} \frac{\partial}{\partial \xi} f\left(\Lambda_{1}, \xi\right) .
\end{aligned}
$$

It is well known that $\lim f\left(\Lambda_{1}, \xi\right)$ exists if $s_{1}(\varepsilon)$ exists [2], and that $s_{\Lambda_{1}}(\xi)$ is bounded by a finite function $p(\xi)$ [4] (see also Appendix D). Let $s_{0}$ be given by $s_{0}=\sup _{\xi \in\left(\xi_{1}, \xi_{2}\right)} p(\xi)$, thus

Consider

$$
s_{\Lambda_{1}}(\xi) \leqq s_{0}, \quad \xi \in\left(\xi_{1}, \xi_{2}\right) .
$$

therefore,

$$
\begin{aligned}
V\left(\Lambda_{1}\right) s_{\Lambda_{1}}(\xi) & =-\xi^{2} \frac{\partial}{\partial \xi} \frac{\log Q\left(\Lambda_{1}, \xi\right)}{\xi} \\
& =\log Q\left(\Lambda_{1}, \xi\right)-\frac{\xi}{Q\left(\Lambda_{1}, \xi\right)} \frac{\partial}{\partial \xi} Q\left(\Lambda_{1}, \xi\right) ;
\end{aligned}
$$

$$
Q\left(\Lambda_{1}, \xi\right) V\left(\Lambda_{1}\right) s_{\Lambda_{1}}(\xi)=\sum_{r}\left(\log Q\left(\Lambda_{1}, \xi\right)+\xi E_{1 r}\right) e^{-\xi E_{1 r}} .
$$

The terms on the right hand side are positive, because $Q\left(\Lambda_{1}, \xi\right)>e^{-\xi E_{1} \text {. }}$. Restricting the summation to $E_{1 r}>\tilde{E}\left(\Lambda_{1}, \alpha\right)$ we get

$$
Q\left(\Lambda_{1}, \xi\right) V\left(\Lambda_{1}\right) s_{\Lambda_{1}}(\xi) \geqq\left(\log Q\left(\Lambda_{1}, \xi\right)+\xi \tilde{E}(\Lambda, \alpha)\right) Q_{\tilde{E}(\Lambda, \alpha)}\left(\Lambda_{1}, \xi\right) .
$$

Furthermore,

$$
Q\left(\Lambda_{1}, \xi\right) \geqq \sum_{E \leqq \tilde{E}(\Lambda, \alpha)} e^{-\xi E_{1 r}} \geqq \Omega_{1}\left(\Lambda_{1}, \tilde{E}\left(\Lambda_{1}, \alpha\right)\right) e^{-\xi \tilde{E}\left(\Lambda_{1}, \alpha\right)},
$$

$\log Q(\Lambda, \xi) \geqq \log \Omega_{1}\left(\Lambda, \tilde{E}\left(\Lambda_{1}, \alpha\right)\right)-\xi \tilde{E}\left(\Lambda_{1}, \alpha\right)=S_{1}\left(\Lambda_{1}, \tilde{E}\left(\Lambda_{1}, \alpha\right)\right)-\xi \tilde{E}\left(\Lambda_{1}, \alpha\right)$

According to the choice of $\tilde{E}\left(\Lambda_{1}, \alpha\right)$ in Lemma 4.2, we have

$$
\begin{gathered}
S_{1}\left(\Lambda_{1}, \tilde{E}\left(\Lambda_{1}, \alpha\right)\right)=V\left(\Lambda_{1}\right) \frac{s_{0}}{\alpha}, \\
\log Q(\Lambda, \xi) \geqq \frac{V\left(\Lambda_{1}\right)}{\alpha} s_{0}-\xi \tilde{E}\left(\Lambda_{1}, \alpha\right) .
\end{gathered}
$$


Inserting (43) into (42) we find

$$
Q\left(\Lambda_{1}, \xi\right) V\left(\Lambda_{1}\right) s_{\Lambda_{1}}(\xi) \geqq \frac{V\left(\Lambda_{1}\right)}{\alpha} s_{0} Q_{\tilde{E}\left(\Lambda_{1}, \alpha\right)},
$$

hence, according to (41),

$$
Q\left(\Lambda_{1}, \xi\right)^{-1} Q_{\tilde{E}\left(\Lambda_{1}, \alpha\right)}\left(\Lambda_{1}, \xi\right) \leqq \alpha \quad \text { if } \quad \xi \in\left(\xi_{1}, \xi_{2}\right)
$$

Since $V\left(\Lambda_{1}\right)^{-1} E_{1}\left(\Lambda_{1}, V\left(\Lambda_{1}\right) \sigma_{1}\right) \rightarrow \varepsilon_{1}\left(\sigma_{1}\right)$, where $\varepsilon_{1}(\sigma)$ is the inverse function of $s_{1}(\varepsilon)$, we have

$$
\theta_{2}\left(\Lambda_{1}\right) \equiv V\left(\Lambda_{1}\right)^{-1} \tilde{E}\left(\Lambda_{1}, \alpha\right)-\varepsilon_{1}\left(\frac{s_{0}}{\alpha}\right) \underset{\Lambda_{1} \rightarrow \infty}{\longrightarrow} 0,
$$

and thus

$\ell\left(\mathscr{I}_{2}\right)=\tilde{E}\left(\Lambda_{1}, \alpha\right)-E_{m} \leqq V\left(\Lambda_{1}\right)\left(\varepsilon_{1}\left(\frac{s_{0}}{\alpha}\right)+\theta_{2}\left(\Lambda_{1}\right)-\varepsilon_{10}\right) \leqq$ const $\kappa_{1} V(\Lambda)$.

This is the point where the necessity of taking the limit $\kappa_{1} \rightarrow 0$ arises: The length $\ell\left(I_{\delta, \kappa_{1}}\right)$ of $I_{\delta, \kappa_{1}}$ [defined in (30)] decreases, if $\delta \rightarrow 0$ as is necessary in order to have $V(\Lambda) \delta \rightarrow 0$. Therefore, the length of $\mathscr{I}\left(\delta, \kappa_{1}, \Lambda\right)$ which is given approximately by $V(\Lambda) \ell\left(I_{\delta, \kappa_{1}}\right)$ increases more slowly than $V(\Lambda)$; [however, an increase of $\ell\left(\mathscr{I}\left(\delta, \kappa_{1}, \Lambda\right)\right)$ can be achieved]. If we want $\ell\left(\mathscr{I}\left(\delta, \kappa_{1}, \Lambda\right)\right)$ grow faster than const $\cdot \kappa_{1} V(\Lambda)$, we have to take the limit $\kappa_{1} \rightarrow 0$.

By definition of $\mathscr{I}\left(\delta, \kappa_{1}, \Lambda\right), E_{m} \in \mathscr{I}\left(\delta, \kappa_{1}, \Lambda\right)$. Let us write $\mathscr{I}\left(\delta, \kappa_{1}, \Lambda\right)$ as $\mathscr{I}\left(\delta, \kappa_{1}, \Lambda\right)=\mathscr{I}^{+}\left(\delta, \kappa_{1}, \Lambda\right) \cup \mathscr{I}^{-}\left(\delta, \kappa_{1}, \Lambda\right)$ with

$$
\begin{aligned}
& \mathscr{I}^{+}\left(\delta, \kappa_{1}, \Lambda\right)=\mathscr{I}\left(\delta, \kappa_{1}, \Lambda\right) \cap\left\{E^{\prime} ; E^{\prime} \geqq E_{m}\right\} ; \\
& \mathscr{I}^{-}\left(\delta, \kappa_{1}, \Lambda\right)=\mathscr{I}\left(\delta, \kappa_{1}, \Lambda\right) \cap\left\{E^{\prime} ; E^{\prime} \leqq E_{m}\right\} .
\end{aligned}
$$

If we can show that

$$
\ell\left(\mathscr{I}^{-}\right) \geqq \ell\left(\mathscr{I}_{1}\right), \quad \ell\left(\mathscr{I}^{+}\right) \geqq \ell\left(\mathscr{I}_{2}\right),
$$

then we know that $\mathscr{I}_{1} \cup \mathscr{I}_{2}$ is covered by $\mathscr{I}\left(\delta, \kappa_{1}, \Lambda\right)$. We divide $I_{\delta, \kappa_{1}}$ in a corresponding manner:

$$
I_{\delta, \kappa_{1}}=I_{\delta, \kappa_{1}}^{+} \cup I_{\delta, \kappa_{1}}^{-}, \quad I^{ \pm}=I \cap\left\{\varepsilon_{2} ; \varepsilon_{2} \gtreqless \tilde{\varepsilon}\left(\kappa_{1}\right)\right\} .
$$

Denote the lengths of $I_{\delta, \kappa_{1}}^{ \pm}$by $\ell_{ \pm}\left(\delta, \kappa_{1}\right)$, then

$$
\ell\left(\mathscr{I}^{ \pm}\left(\delta, \kappa_{1}, \Lambda\right)\right)=\kappa_{2} V(\Lambda) \ell_{\mp}\left(\delta, \kappa_{1}\right) .
$$

Lemma 4.3. For both signs,

$$
\delta^{-1} \inf _{0 \leqq \kappa_{1} \leqq \frac{1}{2}} \ell_{ \pm}\left(\delta, \kappa_{1}\right) \underset{\partial \rightarrow 0}{\longrightarrow} \infty .
$$


Proof. From Lemma 3.3 we conclude that, for $0 \leqq \kappa_{1} \leqq \frac{1}{2}, \tilde{\varepsilon}\left(\kappa_{1}\right)$ is contained in a finite closed interval; application of Lemma B2 of Appendix $\mathrm{B}$ then yields the desired result.

Therefore, we can find a function $v(\delta)$ with

$$
v(\delta) \underset{\delta \rightarrow 0}{\longrightarrow} \infty, \quad \delta v(\delta) \underset{\delta \rightarrow 0}{\longrightarrow} 0, \quad v(\delta) \ell_{0}(\delta) \rightarrow \infty,
$$

where $\ell_{0}(\delta)=\min \left(\inf \ell_{+}\left(\delta, \kappa_{1}\right), \inf \ell_{-}\left(\delta, \kappa_{1}\right)\right)$. Define $\delta_{\Lambda}$ by $v\left(\delta_{\Lambda}\right)=V(\Lambda)$, then

$$
\begin{aligned}
V(\Lambda) \delta_{\Lambda} \underset{\Lambda \rightarrow \infty}{\longrightarrow} 0, \quad \ell\left(\mathscr{I}^{ \pm}\left(\delta_{\Lambda}, \kappa_{1}, \Lambda\right)\right) & =\kappa_{2} V(\Lambda) \ell_{\mp}\left(\delta_{\Lambda}, \kappa_{2}\right) \\
& \geqq \frac{1}{2} V(\Lambda) \ell_{0}\left(\delta_{\Lambda}\right) \underset{\Lambda \rightarrow \infty}{\longrightarrow} \infty,
\end{aligned}
$$

uniformly in $\kappa_{1}, 0 \leqq \kappa_{1} \leqq \frac{1}{2}$. Now we have to choose $\kappa_{1}$ as a function $\kappa_{A}$ of $\Lambda$ in such a way that

$$
\kappa_{\Lambda} \underset{\Lambda \rightarrow \infty}{\longrightarrow} 0, \quad V\left(\Lambda_{1}\right)=\kappa_{\Lambda} V(\Lambda) \underset{\Lambda \rightarrow \infty}{\longrightarrow} \infty, \quad \frac{\kappa_{\Lambda}}{\ell_{0}\left(\delta_{\Lambda}\right)} \underset{\Lambda \rightarrow \infty}{\longrightarrow} 0 .
$$

This is always possible because $V(\Lambda) \ell_{0}\left(\delta_{\Lambda}\right) \rightarrow \infty$. Consequently,

$$
\begin{aligned}
\frac{\ell\left(\mathscr{I}_{1}\right)}{\ell\left(\mathscr{I}^{-}\left(\delta_{\Lambda}, \kappa_{\Lambda}, \Lambda\right)\right)} \leqq \frac{\text { const } \kappa_{A}}{\ell_{0}\left(\delta_{\Lambda}\right)} \underset{\Lambda \rightarrow \infty}{\longrightarrow} 0, \\
\frac{\ell\left(\mathscr{I}_{2}\right)}{\ell\left(\mathscr{I}^{+}\left(\delta_{\Lambda}, \kappa_{\Lambda}, \Lambda\right)\right)} \leqq \frac{{\text { const' } \kappa_{A}}_{\ell_{0}\left(\delta_{\Lambda}\right)}^{\longrightarrow} \rightarrow \rightarrow \infty}{\longrightarrow} 0,
\end{aligned}
$$

and (45) is fulfilled for sufficiently large $\Lambda$; this in turn implies (38), if we insert $\kappa_{\Lambda}$ and $\delta_{\Lambda}$. Now we define $\xi_{\Lambda_{1}}$ by $\xi\left(\kappa_{\Lambda}\right)$ whenever $V\left(\Lambda_{1}\right)=\kappa_{A} V(\Lambda)$. According to Lemma 3.4,

$$
\xi_{A_{1}} \rightarrow \beta=s_{2}^{\prime}-(\varepsilon) \text { or } s_{2+}^{\prime}(\varepsilon)
$$

depending on the case under consideration. Combining the above results with Eq. (36), we finally arrive at

$$
K_{\Lambda} e^{-\alpha_{1}}\left(\omega_{\Lambda_{1}}^{\xi_{\Lambda_{1}}}(A)-\|A\| \alpha\right) \leqq \omega_{\Lambda}^{E}(A \otimes \mathbf{1}) \leqq K_{\Lambda} \omega_{A_{1}}{ }^{\xi_{1}}(A),
$$

where $\alpha_{1}$ and $\alpha$ are positive numbers which can be chosen arbitrarily small, if $\Lambda$ is sufficiently large.

With $A=1$ we get

$$
K_{\Lambda} e^{-x_{1}}(1-\alpha) \leqq 1 \leqq K_{\Lambda}
$$

hence $K_{\Lambda} \underset{\Lambda \rightarrow \infty}{\longrightarrow} 1$. According to the assumptions of Theorem 4.1, $\lim _{A \rightarrow \infty} \omega_{\Lambda}^{E}(A \otimes 1)$ exists, yielding $\omega_{\varepsilon}^{\kappa_{1}=0}(A \otimes 1)$, if we couple $\Lambda_{1}$ and $\Lambda_{2}$ in the above way. Again using (48), we conclude that $\lim _{\Lambda_{1} \rightarrow \infty} \omega_{\Lambda_{1}}^{{ }_{A_{1}}}(A)$ exists and equals $\omega_{\varepsilon}^{\kappa_{1}=0}(A \otimes \mathbf{1})$, which completes the proof of our theorem. 
Remark 4.4. At a first glance, it may seem that the choice of the tangent at $\tilde{\varepsilon}\left(\kappa_{1}\right)$ in seeking a bound for $s_{2}$ is arbitrary. One might think of taking directly the tangent at $\varepsilon$, if $s_{2}^{\prime}(\varepsilon)$ and $s_{2}^{\prime}-(\varepsilon)$ happen to coincide. But then one encounters difficulties in showing that $\mathscr{I}_{1} \cup \mathscr{I}_{2}$ is covered by $\mathscr{I}\left(\delta, \kappa_{1}, \Lambda_{2}\right)$. A comparison of their lengths is no longer an argument since one can no longer exhibit in an easy way a common point of $\mathscr{I}_{1} \cup \mathscr{I}_{2}$ and $\mathscr{I}\left(\delta, \kappa_{1}, \Lambda_{2}\right)$.

\section{Concluding Remarks}

For the study of the equilibrium of two systems, the special kind of the heat transferring wall and, equivalently, of the energy transferring interaction does not matter as long as one does not ask questions about how and when the equilibrium is reached. Therefore, it is a legitimate procedure to make suitable assumptions, as for instance $(\mathrm{C})$, about the interaction in order to arrive at Proposition 2.1. The same arguments yield at least some plausibility that it is reasonable to assume a similar proposition for the microcanonical state, which amounts to starting with $\lambda=0$, as we did in Section IV. We consider this to be the point which to improve is most desirable; but in order to do so, one would have to exhibit more or less general conditions entraining the existence of the limit $\lim _{\Lambda \rightarrow \infty} \omega_{\Lambda}{ }^{E}-$ which, of course, is a hard task -, or confine oneself to fairly simple models.

Given the statement of Proposition 2.1, the proof of Theorem 2.3 does not depend on whatever assumptions are made in order to guarantee the existence of $s(\varrho, \varepsilon)$. Of course, one cannot do without the assumption of stability of the Hamiltonian itself, which is a basic one. It was also used to conclude that the summations in the expressions for $\Omega^{-}(E)$ are finite.

The same holds true of Theorem 4.1. For its proof we used Theorem 2.3 and the properties of the entropy density as a function of the energy density. As was pointed out in Remark 2.6, Theorem 2.3 also holds for systems with different densities $\varrho_{1}, \varrho_{2}$. In Sections III and IV, the densities enter as fixed parameters only, and consequently, we can state Theorem 4.1 for different densities, too. If we are solely interested in system $\mathscr{S}_{1}$, and not in the special kind of heat reservoir, we can omit the case not covered by Theorem 4.1: Case $(\varepsilon)$ with $s_{2}{ }^{\prime}(\varepsilon) \neq s_{2}{ }_{-}^{\prime}(\varepsilon)$, e.g. by a suitable change of $\varrho_{2}$.

In view of the fact that $\mathscr{P}_{1}$ may undergo a phase transition at the temperature $\beta^{-1}$, it is a satisfactory feature of Theorem 4.1 , that the resulting "canonical" state is defined as a limit of Gibbs states with converging temperatures $\xi_{\Lambda_{1}}^{-1}: \xi_{\Lambda_{1}}^{-1} \rightarrow \beta^{-1}$ either from above or below, depending on the case in question, if we temporarily disregard case $(\varepsilon)$. 
Given the possibility of a phase transition, this is precisely the procedure which one expects to result in a well defined state. This leads to the conjecture that there are two different limiting states describing pure phases, corresponding to the cases $\left(\varepsilon_{1}\right)$ and $\left(\varepsilon_{2}\right)$. Then one may ask how the mixture states could emerge. To answer this question, a good guess would be that these states can come out in the case $(\varepsilon)$ when $g_{\kappa_{1}}\left(\varepsilon^{\prime}\right)$ [defined in (17)] has a plateau. We then may, but are not forced to, take $\varepsilon_{m}\left(\kappa_{1}\right)=\kappa_{1} \varepsilon$, and, according to the actually chosen sequence, one could possibly get mixture states.

There is a conjecture stating that

$$
\lim _{\Lambda \rightarrow \infty} \frac{\operatorname{Tr} \delta^{\Lambda}(H(\Lambda)-E) A}{\operatorname{Tr} \delta^{\Lambda}(H(\Lambda)-E)}=\lim _{\Lambda \rightarrow \infty} \frac{\operatorname{Tr} e^{-\beta H(\Lambda)} A}{\operatorname{Tr} e^{-\beta H(\Lambda)}},
$$

if one takes $\beta=\frac{\partial}{\partial \varepsilon} s(\varrho, \varepsilon)=\frac{\partial}{\partial \varepsilon} \lim _{\Lambda \rightarrow \infty} V(\Lambda)^{-1} \log \delta^{\Lambda}(H(\Lambda)-V(\Lambda) \varepsilon)$.

Proving this would be the most direct way of showing the equivalence of microcanonical and canonical ensembles. But one has to assume that $s_{+}^{\prime}(\varepsilon)=s_{-}^{\prime}(\varepsilon)$ and that no phase transition occurs at the temperature $\beta^{-1}$. Moreover, such a proof would certainly require more detailed assumptions about the underlying observable algebra than those employed in the above treatment.

Acknowledgement. The main part of this work was done in Göttingen. I am indebted to Professor Borchers for a series of stimulatıng discussions. Furthermore, I wish to thank Professors Hugenholtz and Winnink for their hospitality and for helpful critical questions.

\section{Appendix A}

We want to give two simple examples of how assumption $(C)$ of Section II can be fulfilled.

1. Consider a system $\mathscr{S}$ with stable $U$ given by tempered potentials $\phi^{k}$. If we divide $\mathscr{S}$ into two parts restricting one group of variables, say $\left\{x_{i}\right\}$, to $\Lambda_{1}$, the other, say $\left\{y_{j}\right\}$, to $\Lambda_{2}$, and define

$$
\begin{gathered}
U_{1}=\sum_{k} \sum_{\ldots} \phi^{k}\left(x_{i_{1}} \ldots x_{i_{k}}\right), \quad U_{2}=\sum_{k} \sum_{\ldots} \phi^{k}\left(y_{J_{1}} \ldots y_{J_{k}}\right), \\
W\left(x_{1} \ldots x_{n_{1}}, y_{1} \ldots y_{n_{2}}\right)=U\left(x_{1} \ldots x_{n_{1}}, y_{1} \ldots y_{n_{2}}\right)-U\left(x_{1} \ldots x_{n_{1}}\right)-U\left(y_{1} \ldots y_{n_{2}}\right),
\end{gathered}
$$

then, of course, $(C)$ is fulfilled.

2. Let us suppose

(C') (i) $W$ is a symmetric operator given by tempered potentials.

(ii) $W$ is relatively bounded with respect to $U_{0}(\Lambda, n)=U_{1}\left(\Lambda_{1}, n_{1}\right)$ $+U_{2}\left(\Lambda_{2}, n_{2}\right), n=n_{1}+n_{2}$ :

$$
\|W(\Lambda, n) \psi\| \leqq a\|\psi\|+b\left\|U_{0}(\Lambda, n) \psi\right\|, \quad \psi \in \mathscr{H}(\Lambda),
$$

with constants $a, b$ which are independent of $\Lambda$ and $n, b<1$. 
Now we use a theorem of Kato ([5], Theorem V.4.11), which gives a lower bound $\gamma_{U_{0}+W}$ of $U_{0}+W$ :

$$
\begin{aligned}
\gamma_{U_{0}+W} & \geqq \gamma_{U_{0}}-\max \left(\frac{a}{1-b}, a+b\left|\gamma_{U_{0}}\right|\right) \\
& =-n B-\max \left(\frac{a}{1-b}, a+b n B\right) \\
& \geqq-n B(2+b) \text { for sufficiently large } n,
\end{aligned}
$$

thus demonstrating that $U_{0}+W$ is stable.

\section{Appendix B}

In this appendix, $f(x)$ always denotes a continuous, concave function of the real variable $x$. According to [3], the left and right derivatives $f_{-}^{\prime}(x)$ and $f_{+}^{\prime}(x)$ exist everywhere, and $f_{+}^{\prime}(x) \leqq f_{-}^{\prime}(x)$.

In addition, one can easily establish the following properties which are not listed in [3]:

\section{Lemma B 1.}

(i) If $x_{1}<x_{2}, a>0$, then $f\left(x_{2}+a\right)-f\left(x_{1}+a\right) \leqq f\left(x_{2}\right)-f\left(x_{1}\right)$.

(ii) If $x_{1}<x_{2}$, then $f_{+}^{\prime}\left(x_{2}\right) \leqq f_{-}^{\prime}\left(x_{2}\right) \leqq f_{+}^{\prime}\left(x_{1}\right) \leqq f_{-}^{\prime}\left(x_{1}\right)$.

(iii) If $x_{1}<x_{2}<x_{3}$, then

and

$$
\frac{f\left(x_{3}\right)-f\left(x_{1}\right)}{x_{3}-x_{1}} \geqq \frac{f\left(x_{3}\right)-f\left(x_{2}\right)}{x_{3}-x_{2}} \geqq f_{-}^{\prime}\left(x_{3}\right),
$$

$$
\frac{f\left(x_{3}\right)-f\left(x_{1}\right)}{x_{3}-x_{1}} \leqq \frac{f\left(x_{2}\right)-f\left(x_{1}\right)}{x_{2}-x_{1}} \leqq f_{+}^{\prime}\left(x_{1}\right) \text {. }
$$

(iv) $f_{-}^{\prime}(x)$ is continuous from the left, $f_{+}^{\prime}(x)$ is continuous from the right.

(v) If $\xi>0$, then $\lim _{\xi \rightarrow 0} f_{+}^{\prime}(x-\xi)=f_{-}^{\prime}(x), \lim _{\xi \rightarrow 0} f_{-}^{\prime}(x+\xi)=f_{+}^{\prime}(x)$.

(vi) Let I be a closed finite interval. Then the limits

and

$$
\lim _{\delta \rightarrow 0} \frac{1}{\delta}(f(x)-f(x-\delta))=f_{-}^{\prime}(x)
$$

$$
\lim _{\delta \rightarrow 0} \frac{1}{\delta}(f(x+\delta)-f(x))=f_{+}^{\prime}(x)
$$

are uniform in $x \in I$.

Proof. (i) Assume $x_{1}<x_{2}<x_{3}$; concavity of $f$ may be expressed as

$$
f\left(x_{2}\right) \geqq \frac{x_{3}-x_{2}}{x_{3}-x_{1}} f\left(x_{1}\right)+\frac{x_{2}-x_{1}}{x_{3}-x_{1}} f\left(x_{3}\right) .
$$

Twofold application of (B1) to $x_{1}<x_{2}<x_{2}+a$ and to $x_{1}<x_{1}+a<x_{2}+a$ yields the desired result. 
(ii) We have only to show that $f_{-}^{\prime}\left(x_{2}\right) \leqq f_{+}^{\prime}\left(x_{1}\right)$. Take a sufficiently small $\delta>0$ and apply (i) to $x_{1}, x_{2}-\delta, a=\delta$ :

$$
\begin{aligned}
f\left(x_{2}\right)-f\left(x_{1}+\delta\right) & \leqq f\left(x_{2}-\delta\right)-f\left(x_{1}\right), \\
\frac{1}{\delta}\left(f\left(x_{2}\right)-f\left(x_{2}-\delta\right)\right) & \leqq \frac{1}{\delta}\left(f\left(x_{1}+\delta\right)-f\left(x_{1}\right)\right) .
\end{aligned}
$$

Taking the limit $\delta \rightarrow 0$ we get $f_{-}^{\prime}\left(x_{2}\right) \leqq f_{+}^{\prime}\left(x_{1}\right)$.

(iii) Due to (B1), we have

$$
\begin{aligned}
\frac{f\left(x_{3}\right)-f\left(x_{2}\right)}{x_{3}-x_{2}} & \leqq \frac{1}{x_{3}-x_{2}}\left(f\left(x_{3}\right)-\text { r.h.s. of }(\mathrm{B} 1)\right) \\
& =\frac{f\left(x_{3}\right)-f\left(x_{1}\right)}{x_{3}-x_{1}}
\end{aligned}
$$

hence $\frac{1}{\delta}\left(f\left(x_{3}\right)-f\left(x_{3}-\delta\right)\right)$ decreases monotonically as $\delta \rightarrow 0$, finally reaching the limit value $f_{-}^{\prime}\left(x_{3}\right)$.

The second part is proved analogously.

(iv) We consider $x<x_{0}, x \rightarrow x_{0}$. According to (ii), $f_{-}^{\prime}(x)$ decreases monotocially for $x \rightarrow x_{0}$, therefore exists $h\left(x_{0}\right)=\lim _{x \rightarrow x_{0}} f_{-}^{\prime}(x)$, and clearly,

$$
f_{-}^{\prime}(x) \geqq h\left(x_{0}\right) \geqq f_{-}^{\prime}\left(x_{0}\right) \text { for } \quad x<x_{0} .
$$

Assume $h\left(x_{0}\right)>f_{-}^{\prime}\left(x_{0}\right)$. Then we can find a $\delta>0$ such that

$$
h\left(x_{0}\right)>\frac{f\left(x_{0}\right)-f\left(x_{0}-\delta\right)}{\delta} \geqq f_{-}^{\prime}\left(x_{0}\right) .
$$

The second inequality is due to (iii). Moreover, due to the continuity of $f$, we can find a $\delta_{1}$ with

$$
h\left(x_{0}\right)>\frac{f\left(x_{0}-\delta_{1}\right)-f\left(x_{0}-\delta-\delta_{1}\right)}{\delta} \geqq \frac{f\left(x_{0}\right)-f\left(x_{0}-\delta\right)}{\delta} .
$$

The second inequality is due to (i). But again according to (iii),

$$
h\left(x_{0}\right)>\frac{1}{\delta}\left(f\left(x_{0}-\delta_{1}\right)-f\left(x_{0}-\delta_{1}-\delta\right)\right) \geqq f_{-}^{\prime}\left(x_{0}-\delta_{1}\right),
$$

which is in contradiction to (B2). In the same way, we can demonstrate the right continuity of $f_{+}^{\prime}(x)$.

(v) This assertion is a corollary of (iv); $f_{-}^{\prime}(x-\xi) \geqq f_{+}^{\prime}(x-\xi) \geqq f_{-}^{\prime}(x)$ and $\lim _{\xi \rightarrow 0} f_{-}^{\prime}(x-\xi)=f_{-}^{\prime}(x)$ imply $\lim _{\xi \rightarrow 0} f_{+}^{\prime}(x-\xi)=f_{-}^{\prime}(x)$.

(vi) Assume the contrary. Then exist an $\varepsilon_{0}>0$, a sequence $\delta_{v}$, $v=1,2, \ldots$, with $\delta_{v} \rightarrow 0$, and a set $\left\{x_{v}\right\} \subset I$ such that

$$
\frac{1}{\delta_{v}}\left(f\left(x_{v}\right)-f\left(x_{v}-\delta_{v}\right)\right)-f_{-}^{\prime}\left(x_{v}\right)>\varepsilon_{0} .
$$


The left hand side of (B3) can be majorized by

$$
f_{+}^{\prime}\left(x_{v}-\delta_{v}\right)-f_{-}^{\prime}\left(x_{v}\right) \leqq f_{-}^{\prime}\left(x_{v}-\delta_{v}\right)-f_{-}^{\prime}\left(x_{v}\right),
$$

and this expression can be made arbitrarily small because $f_{-}^{\prime}$ is continuous from the left, hence uniformly continuous from the left in the compact interval $I$.

Lemma B 2. Let I be a finite, closed interval on the real axis. For $\delta>0, x_{1} \in I$, define

$$
\begin{gathered}
t^{ \pm}\left(x ; x_{1}\right)=f\left(x_{1}\right)+f_{ \pm}^{\prime}\left(x_{1}\right)\left(x-x_{1}\right), \\
\mathscr{I}\left(\delta ; x_{1}\right)=\left\{x ; f(x)>t^{+}\left(x ; x_{1}\right)-\delta \text { or } f(x)>t^{-}\left(x ; x_{1}\right)-\delta\right\} .
\end{gathered}
$$

Due to the continuity and concavity of $f, \mathscr{I}\left(\delta ; x_{1}\right)$ is an interval $\left(y_{-}\left(\delta ; x_{1}\right)\right.$, $\left.y_{+}\left(\delta ; x_{1}\right)\right)$, with

Define

$$
\begin{array}{lll}
f(x)>t^{-}\left(x ; x_{1}\right)-\delta & \text { for } & x \in\left(y_{-}\left(\delta ; x_{1}\right), x_{1}\right), \\
f(x)>t^{+}\left(x ; x_{1}\right)-\delta & \text { for } & x \in\left(x_{1}, y_{+}\left(\delta ; x_{1}\right)\right) .
\end{array}
$$

$$
\ell_{ \pm}\left(\delta ; x_{1}\right)=\left|y_{ \pm}\left(\delta ; x_{1}\right)-x_{1}\right| \text {. }
$$

Then exists $\ell_{0}{ }^{ \pm}(\delta)=\inf _{x_{1} \in I} \ell_{ \pm}\left(\delta ; x_{1}\right)$ and

$$
\frac{\delta}{\ell_{0}{ }^{ \pm}(\delta)} \underset{\delta \rightarrow 0}{\longrightarrow} 0 .
$$

Proof. The cited properties of $\mathscr{I}\left(\delta ; x_{1}\right)$ are trivial. It suffices to show that $\delta / \ell_{ \pm}\left(\delta ; x_{1}\right)$ converges uniformly in $x_{1}$. This then implies that $\ell_{0}{ }^{ \pm}(\delta) \neq 0$ and $\delta / \ell_{0} \pm \rightarrow 0$.

We shall only give the proof for one sign. Suppose non-uniformity; then there are an $\varepsilon_{0}>0$, a sequence $\delta_{v} \rightarrow 0$ and a set $\left\{x_{v}^{+}\right\} \subset I$ such that

$$
\frac{\delta_{v}}{\ell_{+}\left(\delta_{v} ; x_{v}^{+}\right)} \geqq \varepsilon_{0} .
$$

According to the definition of $\ell_{+}$,

$$
\begin{aligned}
f\left(x_{v}^{+}+\ell_{+}\left(\delta_{v}: x_{v}\right)\right) & =t^{+}\left(x_{v}^{+}+\ell_{+}\left(\delta_{v} ; x_{v}^{+}\right) ; x_{v}\right)-\delta_{v} \\
& =f\left(x_{v}^{+}\right)+f_{+}^{\prime}\left(x_{v}^{+}\right) \ell_{+}\left(\delta_{v} ; x_{v}^{+}\right)-\delta_{v},
\end{aligned}
$$

whence, with $\ell_{+}\left(\delta_{v}, x_{v}^{+}\right) \equiv \ell_{v}$,

$$
\varepsilon_{0} \leqq \frac{\delta_{v}}{\ell_{+}\left(\delta_{v} ; x_{v}^{+}\right)} \leqq f_{+}^{\prime}\left(x_{v}^{+}\right)-\frac{f\left(x_{v}^{+}+\ell_{v}\right)-f\left(x_{v}^{+}\right)}{\ell_{v}} .
$$

As $v \rightarrow \infty, \delta_{v}$ and thus $\ell_{v}$ vanish; and the right hand side approaches zero uniformly in $x_{v}$ aaccording to Lemma B1 (vi), which is a contradiction. 


\section{Appendix C}

Proof of Lemma 3.5. We restrict ourselves to case $\left(\varepsilon_{1}\right)$, the other case can be treated analogously.

(i) Suppose $\kappa_{1}<\kappa_{1}^{\prime}$ and

Then

$$
\eta_{s}\left(\kappa_{1}\right)<\eta_{s}\left(\kappa_{1}^{\prime}\right)
$$

$$
\frac{\kappa_{1}}{1-\kappa_{1}}\left(\eta_{s}\left(\kappa_{1}\right)-\varepsilon\right)<\frac{\kappa_{1}^{\prime}}{1-\kappa_{1}^{\prime}}\left(\eta_{s}\left(\kappa_{1}^{\prime}\right)-\varepsilon\right),
$$

[remember that $\eta_{s}\left(\kappa_{1}\right)>\varepsilon$ according to Lemma 3.4], hence

and thus

$$
\tilde{\varepsilon}_{s}\left(\kappa_{1}\right)>\tilde{\varepsilon}_{s}\left(\kappa_{1}^{\prime}\right),
$$

$$
s_{1+}^{\prime}\left(\eta_{s}\left(\kappa_{1}\right)\right) \leqq s_{2}^{\prime}-\left(\tilde{\varepsilon}\left(\kappa_{1}\right)\right) \leqq s_{2}^{\prime}+\left(\tilde{\varepsilon}_{s}\left(\kappa_{1}^{\prime}\right)\right) \leqq s_{1}^{\prime}-\left(\eta_{s}\left(\kappa_{1}^{\prime}\right)\right) \leqq s_{1}^{\prime}\left(\eta_{s}\left(\kappa_{1}\right)\right) .
$$

[The first and third inequalities are due to the supremum conditions $(22 \mathrm{a}, \mathrm{b})$, the second and fourth ones due to (C2) and (C 1).] (C 3) implies that we can put equality signs instead of the inequality signs. Furthermore,

$$
\begin{aligned}
\tilde{\varepsilon}_{s}\left(\kappa_{1}\right) & \equiv \varepsilon-\frac{\kappa_{1}}{1-\kappa_{1}}\left(\eta_{s}\left(\kappa_{1}\right)-\varepsilon\right)>\varepsilon-\frac{\kappa_{1}}{1-\kappa_{1}}\left(\eta_{s}\left(\kappa_{1}^{\prime}\right)-\varepsilon\right) \\
& \equiv \varepsilon^{*}\left(\kappa_{1}\right)>\tilde{\varepsilon}_{s}\left(\kappa_{1}^{\prime}\right), \\
S_{2}{ }^{\prime}\left(\tilde{\varepsilon}_{s}\left(\kappa_{1}\right)\right) & \leqq S_{2+}^{\prime}\left(\varepsilon^{*}\left(\kappa_{1}\right)\right) \leqq S_{2+}{ }^{\prime}\left(\tilde{\varepsilon}_{s}\left(\kappa_{1}^{\prime}\right)\right) .
\end{aligned}
$$

But due to (C3) with equality signs, we have

$$
s_{2}^{\prime}-\left(\tilde{\varepsilon}_{s}\left(\kappa_{1}\right)\right)=s_{2}^{\prime}+\left(\tilde{\varepsilon}_{s}\left(\kappa_{1}^{\prime}\right)\right),
$$

hence the derivative of $s_{2}$ is constant between $\tilde{\varepsilon}_{s}\left(\kappa_{1}^{\prime}\right)$ and $\tilde{\varepsilon}_{s}\left(\kappa_{1}\right)$, and thus

$$
s_{2}^{\prime}+\left(\tilde{\varepsilon}_{s}\left(\kappa_{1}^{\prime}\right)\right)=s_{2}^{\prime}\left(\varepsilon^{*}\left(\kappa_{1}\right)\right)=s_{2}^{\prime}\left(\varepsilon^{*}\left(\kappa_{1}\right)\right)=s_{2}^{\prime}-\left(\tilde{\varepsilon}_{s}\left(\kappa_{1}\right)\right) .
$$

Combining (C 3) (with equality signs) with (C 5) and (C 1) we get

$$
\begin{aligned}
& s_{1+}^{\prime}\left(\eta_{s}\left(\kappa_{1}^{\prime}\right)\right) \leqq s_{1+}^{\prime}\left(\eta_{s}\left(\kappa_{1}\right)\right)=s_{2}^{\prime}\left(\varepsilon^{*}\left(\kappa_{1}\right)\right), \\
& s_{1}^{\prime}\left(\eta_{s}\left(\kappa_{1}^{\prime}\right)\right)=s_{2+}^{\prime}\left(\varepsilon^{*}\left(\kappa_{1}\right)\right) .
\end{aligned}
$$

But these are the supremum conditions for $\kappa_{1}$, fulfilled by $\eta_{s}\left(\kappa_{1}^{\prime}\right)$, with $\eta_{s}\left(\kappa_{1}^{\prime}\right)>\eta_{s}\left(\kappa_{1}\right)$ in contradiction to the definition of $\eta_{s}\left(\kappa_{1}\right)$ as being maximal. Hence

$$
\eta_{s}\left(\kappa_{1}\right) \geqq \eta_{s}\left(\kappa_{1}^{\prime}\right) \text {. }
$$

(ii) $\tilde{\varepsilon}_{s}\left(\kappa_{1}\right)$ is a continuous function of $\kappa_{1}$ whenever $\eta_{s}\left(\kappa_{1}\right)$ is continuous. Now assume that $\eta_{s}$ has a discontinuity at a point $\kappa_{0}$. Without loss of generality, let us suppose that it is discontinuous from the right: there is an $\alpha>0$ with

$$
\eta_{s}\left(\kappa_{0}+\delta\right)<\eta_{s}\left(\kappa_{0}\right)+\alpha \text { for all } \delta>0 .
$$


This implies a discontinuity for $\tilde{\varepsilon}_{s}\left(\kappa_{1}\right)$ : for sufficiently small $\delta$,

$$
\tilde{\varepsilon}_{s}\left(\kappa_{0}+\delta\right)=\varepsilon-\frac{\kappa_{0}+\delta}{1-\kappa_{0}-\delta}\left(\eta_{s}\left(\kappa_{0}+\delta\right)-\varepsilon\right)>\tilde{\varepsilon}_{s}\left(\kappa_{0}\right)+\alpha^{\prime}, \alpha^{\prime}>0,
$$

i.e. $\tilde{\varepsilon}_{s}\left(\kappa_{1}\right)$ jumps downward as $\kappa_{1}$ decreases to $\kappa_{0}$.

On the other hand, $\tilde{\varepsilon}_{s}\left(\kappa_{1}\right)$ goes to $\varepsilon$ from below as $\kappa_{1} \rightarrow 0$, therefore, there must be increasing parts where $\tilde{\varepsilon}_{s}\left(\kappa_{1}\right)$ has to be continuous. Thus $\tilde{\varepsilon}_{s}\left(\kappa_{1}\right)$ assumes a continuum of values as $\kappa_{1} \rightarrow 0$, and we can find sequences $\kappa_{1 v}$ approaching zero, for which $s_{2}^{\prime}\left(\tilde{\varepsilon}_{s}\left(\kappa_{1 v}\right)\right)=s_{2}^{\prime}\left(\tilde{\varepsilon}_{s}\left(\kappa_{1 v}\right)\right)$ because $s_{2-}^{\prime} \neq s_{2+}^{\prime}$ is only possible for countably many points.

\section{Appendix D}

The exact statement of Griffith, referred to in the proof of Theorem 4.1, is that due to the convergence of the functions $f\left(\Lambda_{1}, \xi\right)$ which are concave functions of $\xi^{-1}$, their derivatives also converge to the derivative of the limit function wherever the latter is continuous:

Lemma (Griffith). Let $g_{k}(x)$ be concave, differentiable functions defined on $[a, b]$, and assume that $\lim g_{k}(x)=g(x)$ exists. Then we have $\lim g_{k}^{\prime}(x)=g^{\prime}(x)$ for all $x$ where $g^{\prime}(x)$ is continuous.

$g(x)$ is also concave, hence $g^{\prime}(x)$ has at most countably many jump discontinuities. $g_{+}^{\prime}$ and $g_{-}^{\prime}$ exist everywhere. One can sharpen the above lemma to get the following

Lemma D 1. Let $g_{k}$ be as above. For all $x \in[a, b]$, we have

$$
g_{+}^{\prime}(x) \leqq \liminf _{k \rightarrow \infty} g_{k}^{\prime}(x) \leqq \limsup _{k \rightarrow \infty} g_{k}^{\prime}(x) \leqq g_{-}^{\prime}(x) .
$$

The proof runs completely analogous to that one of Griffith's Lemma (Appendix A of [6]), if one takes into account that $g_{-}^{\prime}$ is continuous from the left and $g_{+}^{\prime}$ is continuous from the right. Clearly, Lemma D1 implies the existence of a finite upper bound $p(x)$ of $g_{k}^{\prime}(x)$.

\section{Appendix E}

Notations. $d(\Lambda)=$ diameter of $\Lambda=\sup _{x, y \in \Lambda}|x-y|$;

$\Delta\left(\Lambda_{1}, \Lambda_{2}\right)=$ distance of $\Lambda_{1}$ and $\Lambda_{2}=\inf _{x \in \Lambda_{1}, y \in \Lambda_{2}}|x-y|$

$V_{h}(\Lambda)=$ volume of the subset of points of $\Lambda$ with distance less than $h$ to the boundary of $\Lambda$;

$\Lambda \underset{F}{\rightarrow} \infty$ : the regions $\Lambda$ tend to infinity in the sense of Fisher, i.e.

(i) $V(\Lambda) \rightarrow \infty$,

(ii) there exists a function $\pi(\alpha), \alpha \in \mathbb{R}, \alpha<1$, with $\lim _{\alpha \rightarrow 0} \pi(\alpha)=0$,

$$
V_{\alpha d(\Lambda)}(\Lambda) / V(\Lambda) \leqq \pi(\alpha)
$$


Proposition E1. Let $\left\{\Lambda_{1}\right\}$ and $\left\{\Lambda_{2}\right\}$ be sequences of regions in $\mathbb{R}^{v}$, $\Lambda_{i} \underset{F}{\longrightarrow} \infty, i=1,2$. Then $\Lambda=\Lambda_{1} \cup \Lambda_{2} \rightarrow \vec{F}$ if and only if

$$
\Delta\left(\Lambda_{1}, \Lambda_{2}\right) \leqq c\left(d\left(\Lambda_{1}\right)+d\left(\Lambda_{2}\right)\right)
$$

where $c$ is an appropriate positive constant.

To be precise, let us introduce a real parameter $\tau$ and consider $\Lambda_{i}(\tau)$, $\Lambda_{i}(\tau) \vec{F} \infty$ if $\tau \rightarrow \infty, i=1,2$. Then $\Lambda(\tau)=\Lambda_{1}(\tau) \cup \Lambda_{2}(\tau)$ is a well-defined sequence.

Let us define

$$
\gamma(\tau)=\frac{V\left(\Lambda_{1}(\tau)\right)}{V\left(\Lambda_{2}(\tau)\right)}, \quad \varrho(\tau)=\frac{d\left(\Lambda_{1}(\tau)\right)}{d\left(\Lambda_{2}(\tau)\right)} .
$$

We need the following

Lemma E 2. If $\Lambda_{i} \rightarrow \infty, i=1,2$, for $\tau \rightarrow \infty$, then there exist constants $k_{1}, k_{2} \neq 0, \infty$ such that

$$
k_{1} \leqq \frac{\varrho(\tau)^{v}}{\gamma(\tau)} \leqq k_{2}
$$

Proof. $\Lambda \underset{F}{\rightarrow} \infty$ implies the existence of constants $C, C^{\prime}$ such that

$$
C d(\Lambda)^{v} \leqq V(\Lambda) \leqq C^{\prime} d(\Lambda)^{v} .
$$

$\left[V_{\alpha d(\Lambda)}(\Lambda)\right.$ contains a sphere of radius $\frac{1}{2} \alpha d(\Lambda)$, thence the first inequality.] Application of (E 5) to $V\left(\Lambda_{1}\right)$ and $V\left(\Lambda_{2}\right)$ yields

$$
\frac{C_{2}}{C_{1}{ }^{\prime}} \leqq \frac{V\left(\Lambda_{2}\right)}{d\left(\Lambda_{2}\right)^{v}} \cdot \frac{d\left(\Lambda_{1}\right)^{v}}{V\left(\Lambda_{1}\right)} \leqq \frac{C_{2}^{\prime}}{C_{1}},
$$

and hence (E4) with

$$
k_{1}=C_{2} / C_{1}^{\prime}, \quad k_{2}=C_{2}{ }^{\prime} / C_{1} .
$$

Proof of Proposition E1. We first show the necessity of condition (E2). Assume that $\Delta\left(\Lambda_{1}, \Lambda_{2}\right)$ grows faster than $d\left(\Lambda_{1}\right)+d\left(\Lambda_{2}\right)$. Then, for arbitrarily small $\alpha$, we can find a $\Lambda_{0}=\Lambda_{10} \cup \Lambda_{20}$ such that $\Delta\left(\Lambda_{10}, \Lambda_{20}\right)$ $\geqq \frac{1}{\alpha}\left(d\left(\Lambda_{10}\right)+d\left(\Lambda_{20}\right)\right)$, hence

$$
\alpha d\left(\Lambda_{0}\right) \geqq \alpha \Delta\left(\Lambda_{10}, \Lambda_{20}\right) \geqq d\left(\Lambda_{10}\right)+d\left(\Lambda_{20}\right),
$$

and therefore,

$$
V_{\alpha d\left(\Lambda_{0}\right)}\left(\Lambda_{0}\right)=V\left(\Lambda_{10}\right)+V\left(\Lambda_{20}\right)=V\left(\Lambda_{0}\right),
$$

which is in contradiction to the conditions for the Fisher limit. 
Now let us assume that $\Delta\left(\Lambda_{1}, \Lambda_{2}\right) \leqq c\left(d\left(\Lambda_{1}\right)+d\left(\Lambda_{2}\right)\right)$ and thus

$$
\begin{gathered}
d(\Lambda(\tau)) \leqq c^{\prime}\left(d\left(\Lambda_{1}(\tau)\right)+d\left(\Lambda_{2}(\tau)\right)\right) \equiv \theta(\tau), \quad c^{\prime}=c+1, \\
\frac{V_{\alpha d(\Lambda)}(\Lambda)}{V(\Lambda)} \leqq \frac{V_{\alpha \theta(\tau)}\left(\Lambda_{1}\right)+V_{\alpha \theta(\tau)}\left(\Lambda_{2}\right)}{V(\Lambda)} .
\end{gathered}
$$

We consider the following three regions separately $(\alpha<1)$ :

$$
\begin{aligned}
& G_{1}=\left\{(\alpha, \tau) ; \varrho(\tau)>\frac{1}{\sqrt{\alpha}}\right\}, \\
& G_{2}=\left\{(\alpha, \tau) ; \frac{1}{\sqrt{\alpha}} \geqq \varrho(\tau) \geqq \sqrt{\alpha}\right\}, \\
& G_{3}=\{(\alpha, \tau) ; \sqrt{\alpha}>\varrho(\tau)\} .
\end{aligned}
$$
hence

(i) $(\alpha, \tau) \in G_{1}$ : then $d\left(\Lambda_{2}\right) \leqq \sqrt{\alpha} d\left(\Lambda_{1}\right), \alpha \theta(\tau) \leqq \alpha c^{\prime}(1+\sqrt{\alpha}) d\left(\Lambda_{1}\right)$, and

$$
\frac{V_{\alpha \theta(\tau)}\left(\Lambda_{1}\right)}{V(\Lambda)} \leqq \frac{V_{\alpha c^{\prime}(1+\sqrt{\alpha}) d\left(\Lambda_{1}\right)}\left(\Lambda_{1}\right)}{V\left(\Lambda_{1}\right)} \leqq \pi_{1}\left(\alpha c^{\prime}(1+\sqrt{\alpha})\right)
$$

because we suppose $\Lambda_{1} \rightarrow \underset{F}{\infty}$, i.e. the existence of $\pi_{1}(\alpha)$ such that (E1) is fulfilled. Furthermore,

$$
\frac{V_{\alpha \theta(\tau)}\left(\Lambda_{2}\right)}{V(\Lambda)} \leqq \frac{V\left(\Lambda_{2}\right)}{V\left(\Lambda_{1}\right)} \leqq \frac{1}{\gamma(\tau)} \leqq k_{2} \varrho(\tau)^{-v} \leqq k_{2} \alpha^{\frac{v}{2}}
$$

according to the above Lemma. Insertion of (E 8) and (E9) into (E7) yields

$$
\frac{V_{\alpha d(\Lambda)}(\Lambda)}{V(\Lambda)} \leqq \pi^{(1)}(\alpha), \pi^{(1)}(\alpha)=k_{2} \alpha^{\frac{v}{2}}+\pi_{1}\left(\alpha c^{\prime}(1+\sqrt{\alpha})\right)
$$

(ii) $(\alpha, \tau) \in G_{2}$ implies $\frac{1}{\sqrt{\alpha}} d\left(\Lambda_{2}\right) \geqq d\left(\Lambda_{2}\right) \geqq \sqrt{\alpha} d\left(\Lambda_{2}\right)$; therefore

$$
\begin{gathered}
\alpha \theta(\tau) \leqq \alpha c^{\prime}\left(1+\frac{1}{\sqrt{\alpha}}\right) d\left(\Lambda_{1}\right), \\
\alpha \theta(\tau) \leqq \alpha c^{\prime}\left(\frac{1}{\sqrt{\alpha}}+1\right) d\left(\Lambda_{2}\right) ; \\
\frac{V_{\alpha \theta(\tau)}\left(\Lambda_{i}\right)}{V(\Lambda)} \leqq \frac{V_{c^{\prime}(\alpha+\sqrt{\alpha}) d\left(\Lambda_{i}\right)}\left(\Lambda_{i}\right)}{V\left(\Lambda_{i}\right)} \leqq \pi_{i}\left((\alpha+\sqrt{\alpha}) c^{\prime}\right), \quad i=1,2, \\
\frac{V_{\alpha d(\Lambda)}(\Lambda)}{V(\Lambda)} \leqq \pi^{(2)}(\alpha), \quad \pi^{(2)}(\alpha)=\pi_{1}\left((\alpha+\sqrt{\alpha}) c^{\prime}\right)+\pi_{2}\left((\alpha+\sqrt{\alpha}) c^{\prime}\right) .
\end{gathered}
$$


(iii) For $(\alpha, \tau) \in G_{3}$ we have $d\left(\Lambda_{1}\right) \leqq \sqrt{\alpha} d\left(\Lambda_{2}\right)$, thence

and

$$
\begin{aligned}
\alpha \theta(\tau) & \leqq \alpha c^{\prime}(\sqrt{\alpha}+1) d\left(\Lambda_{2}\right), \\
\frac{V_{\alpha \theta(\tau)}\left(\Lambda_{2}\right)}{V(\Lambda)} & \leqq \pi_{2}\left(\alpha c^{\prime}(\sqrt{\alpha}+1)\right),
\end{aligned}
$$

$$
\frac{V_{\alpha \theta(\tau)}\left(\Lambda_{1}\right)}{V(\Lambda)} \leqq \frac{V\left(\Lambda_{1}\right)}{V\left(\Lambda_{2}\right)}=\gamma(\tau) \leqq \frac{\varrho(\tau)^{v}}{k_{1}} \leqq \frac{\alpha^{\frac{v}{2}}}{k_{1}},
$$

which yields

$$
\frac{V_{\alpha d(\Lambda)}(\Lambda)}{V(\Lambda)} \leqq \pi^{(3)}(\alpha), \pi^{(3)}(\alpha)=\frac{\alpha^{\frac{v}{2}}}{k_{1}}+\pi_{2}\left(\alpha c^{\prime}(1+\sqrt{\alpha})\right) .
$$

From (E10), (E11), and (E12), it is clear that

$$
\pi(\alpha) \equiv \max _{i=1,2,3} \pi^{(i)}(\alpha) \underset{\alpha \rightarrow 0}{\longrightarrow} 0,
$$

and

$$
\frac{V_{\alpha d(\Lambda)}(\Lambda)}{\mathrm{V}(\Lambda)} \leqq \pi(\alpha) \text { for all } \alpha<1, \text { i.e. } \Lambda \rightarrow \underset{F}{ } \infty \quad \text { Q.E.D. }
$$

\section{References}

1. Fisher, M.E.: Arch. Rat. Mech. Anal. 17, 377 (1964)

2. Ruelle, D.: Statistical Mechanics. New York: W. A. Benjamin, Inc. 1969

3. Hewitt, E., Stromberg, K.: Real and abstract analysis. Berlin-Heidelberg-New York: Springer 1965

4. Griffiths, R. B.: J. Math. Phys. 6, 1447 (1965)

5. Kato, T.: Perturbation theory for linear operators. Berlin-Heidelberg-New York: Springer 1966

6. Griffiths, R. B.: J. Math. Phys. 5, 1215 (1964)

H. Roos

Institute for Theoretical Physics

P.O. Box 800

Groningen, The Netherlands 